\title{
Sediment transport models in Shallow Water equations and numerical approach by high order finite volume methods.
}

\author{
M. J. Castro Díaz ${ }^{\dagger}$ E. D. Fernández-Nieto ${ }^{\ddagger} \&$ A.M. Ferreiro ${ }^{\S}$
}

January 18, 2008

\begin{abstract}
This paper is concerned with the numerical approximation of bedload sediment transport due to water evolution. For the hydrodynamical component we consider shallow water equations. The morphodynamical component is defined by a continuity equation, which is defined in function of the solid transport discharge. We present several deterministic models, such as Meyer-Peter\&Müller, Van Rijn or Grass model. We also present an unified definition for the solid transport discharge, and we compare with Grass model. Both components define a coupled system of equations that can be re-write as a non-conservative hyperbolic system. To discretize it, we consider finite volume methods with or without flux limiters and high order state reconstructions. Finally we present several tests, where we observe numerically the order of the numerical schemes, Comparisons with analytical solutions and experimental data are also presented.
\end{abstract}

Short title : Numerical approach of sediment transport models.

Keywords : Well-balanced, Finite Volume Method, upwinding, shallow water, source terms, sediment transport.

Subject Classifications : AMS (MOS) : 65N06, 76B15, 76M20, 76N99.

\section{Introduction}

The sediment can be defined as a fragmented material from rocks that has been formed by different physical and/or chemical process. The study of sediment transport processes includes movement of rocks in a mountain as material diffusion in water, among other processes. Transport is caused by gravity effects and by friction effects with the air or the fluid containing the sediment.

Sediment transport is usually divided into three types: bedload, saltation and suspension (see Figure 1). Bedload transport is defined as the type of transport where sediment grains roll or slide along the bed. Saltation transport is defined as the type of transport where single grains jump over the bed a length proportional to their diameter, losing for instants the contact with the soil. Sediment is suspended when the flux is intense enough such as the sediment grains reach height over the bed.

In this paper we face the study of bedload sediment transport. To model bedload sediment transport process caused by the movement of a fluid in contact with the sediment layer, we consider a coupled model constituted by a hydrodynamical component and a morphodynamical component.

\footnotetext{
*This research was partially supported by Spanish Government Research Projects BFM2003-07530-C02-01 and BFM2003-07530-C02-02.

†Dpto. Análisis Matemático, Universidad de Málaga, Campus Teatinos s/n, Málaga, Spain (castro@anamat.cie.uma.es)

†Departamento de Matemática Aplicada I, Universidad de Sevilla. 41012 Sevilla, Spain (edofer@us.es).

$\S$ Dpto. Análisis Ecuaciones Diferenciales y Análisis Numérico, Universidad de Sevilla, C/ Tarfia S/N, Sevilla (anafefe@us.es)
} 


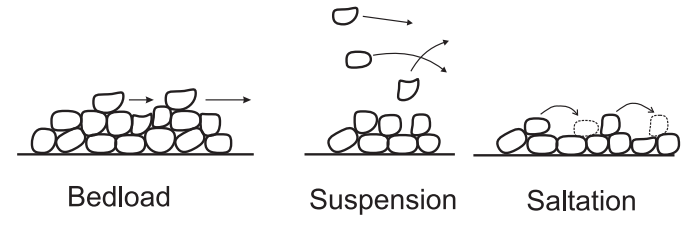

Figure 1: Types of sediment transport.

The hydrodynamical component is modeled by Shallow Water equations, which is are used to study fluid movement in rivers, channel, coast areas, etc, while a sediment transport equation, depending on solid transport flux, is considered to model the morphodynamical component.

In literature different equations to model the solid transport sediment flux could be found (Grass equation [25], Meyer-Peter\&Müller's equation [41], Van Rijn's equation [58]-[60], Nielsen's equation [42], Kalinske ([34], [35]), Einstein's equation ([18], [19], etc), generally obtained by empirical methods. Among all these formulae, some are deterministic formulae and others are based on probabilistic terms. In this paper we only consider deterministic equations (see Section 2.3).

In most of them, except for Grass model, the movement of the sediment is controlled by a physical parameter called critical shear stress, usually determined experimentally.

Therefore, hydrodynamical and morphodynamical components constitute a coupled system, with three unknowns: $h(x, t)$, the thickness of the fluid, $q(x, t)$, the discharge of the fluid and $z_{b}(x, t)$, the thickness of the sediment layer. This coupled system can be written as a non-conservative hyperbolic system

$$
\frac{\partial W}{\partial t}+\mathcal{A}(W) \frac{\partial W}{\partial x}=0
$$

It is well known that standard methods that solve correctly systems of conservation laws can fail in solving systems of balance laws, specially when approaching equilibria or near to equilibria solutions. Moreover, they can produce unstable methods when they are applied to coupled systems of conservation or balance laws. Many authors have studied well-balanced numerical schemes for balance laws or coupled systems. See [50], [4], [26], [27], [40], [22], [23], [46], [9], [56], [47], [12], [13], [3], [6], $[43] \ldots$

One of these techniques consists on discretizing the conservative terms of the system by using a well-known solver for homogeneous conservative systems and studying a discretization of the source term and/or the non-conservative terms. On related papers authors study if with the proposed discretization the numerical scheme exactly or approximately preserves a given stationary solution or a family of equilibria. The form in which the discretization of the source term and/or the coupled term is discretized depends, in general, on the specific considered system, and, usually, the extension to high order numerical schemes becomes a difficult task. In [12] it was shown that the technique of Modified Equations can be helpful in this procedure.

For example, this technique has been used to extend Roe method, Lax-Wendrof with flux-limiters or HLL scheme among other classical schemes. Roe method was studied in [4] and [9], a family of numerical schemes, including numerical schemes using flux-limiters, was studied in [13]. In [36] Kurganov and Levy extend HLL scheme with state reconstruction for Shallow Water equation with topography as source term, using the water surface and the discharge as unknowns. This method is known as central-upwind. In [20] Fraccarollo et al extend HLL method for a system coupling Shallow Water equation with a sediment layer in order to study intense sediment transport. They introduce a lateralized discretization of the coupled term, which consists on different approximations to the left and to the right. They also write the system in terms of the water surface. They call this method LHLL.

Another technique consists on considering balance laws or coupled systems as a particular case of the one-dimensional quasilinear hyperbolic system (1). Once the system is rewritten under this form, 
piecewise constant approximations of the solutions are considered, which are updated by means of Approximate Riemann Solvers at the intercells. . The extension to high order numerical schemes is in this case straightforward.

Following [44], this second procedure has been considered in this work. The main difficulty both from the mathematical and the numerical points of view comes from the presence of non-conservative products, which makes difficult even the definition of weak solutions. Many papers have been devoted to the definition and the stability of non-conservative products and its application to the definition of weak solutions of non-conservative hyperbolic systems: see [63], [11], [15], [16], [48], [7], [38], [5], [1] ...

In this article we assume the definition of non-conservative products as Borel measures given by Dal Maso, LeFloch, and Murat in [16]. This definition, which depends on the choice of a family of paths in the phase space, allows to give a rigorous definition of weak solutions of (1). Together with the definition of weak solutions, a notion of entropy has to be chosen, as the usual Lax's concept or one related to an entropy pair. The classical theory of simple waves of hyperbolic systems of conservation laws and the results concerning the solutions of Riemann problems can be then extended to systems (1).

The choice of the family of paths may be, in general, a difficult task. The introduction of such family of paths also allows to give a general definition of Roe linearizations for non-conservative systems. In [43] the authors propose a general definition of well-balanced schemes to solve systems like (1).

J. Hudson in [31] proposed several numerical approximations of a system constituted by shallow water equations and bedload sediment transport equation using Grass model. He considers different reformulations of the problem.

E. Peña González in [45] proposed a numerical approximation of a non-coupled system formed by shallow water equations and bedload sediment transport equation. He applies a finite volumes scheme based on Roe scheme to obtain the shallow water unknowns, thickness and discharge of the fluid. The Approximated velocity and thickness of the fluid are used in bedload sediment transport equation to get an approximation of thickness of the sediment layer. He also proposed different techniques of measurement of the thickness of the sediment layer. In Section 6.3 a comparison with one of the experiments performed in his work is shown.

In [20] a second order LHLL method for the computation of open channel flows in conditions of rapid bed erosion and intense sediment transport is presented. The phenomena that govern these flows are different from those presented in bedload sediment transport, where the interaction between the fluid and the sediment is very weak.

One of the main difficulty to obtain a precise numerical approximation for bedload sediment transport models is that the interaction between fluid and sediment is usually very weak (see Section 2.5) and long time simulations are needed. For this reason, as characteristic velocities of fluid and sediment are very different, low order numerical schemes are very diffusive (see Section 6.1). So, it is necessary to use high order numerical schemes, at least bigger than two to obtaining precise results. In this paper we consider numerical schemes based on flux limiters and numerical schemes based on state reconstructions for non-conservative hyperbolic systems (see [10]).

This paper is organized as follows: In Section 2 we present the system of equations for different morphodynamical models, such as Meyer-Peter\&Müller, Van Rijn or Grass. In Subsection 2.4 we present a unified formulation of the presented models, redefining the solid transport discharge. In Subsection 2.5 we perform a comparison with Grass model when the critical shear stress is set to zero, studying the type of interaction between the fluid and the sediment. In Subsection 2.6 we present the coupled system of equations. We introduce a new variable that allows us to simplify the system. We re-write it as a non-conservative hyperbolic system. In Section 3 we present finite volume methods for non-conservative hyperbolic systems with and without flux-limiters. In Section 4 we present high order finite-volume methods using state reconstructions, concretely we have used WENO reconstructions. In Section 5 we present Roe matrix for the different considered models, in an approximated way, and how we treat the problem of transition cells: transition with-without sediment and transition withwithout movement. Finally in Section 6 we present three numerical tests, we observe numerically the 
order of the different numerical schemes, we also compare with an analytical solution for Grass model and finally we compare with experimental data.

\section{Sediment transport models in Shallow Water equations}

In this section we present a system of equations that models sediment transport in areas such us rivers, lakes or coast. To solve the problem of sediment transport by a fluid, a coupled mathematical model can be used with a hydrodynamical component and a morphodynamical component.

- For the hydrodynamical component we consider Shallow Water equations, which simulates the movement of a fluid in rivers, channels, coastal areas, etc. (See [56], [9]),

- The morphodynamical component is described with a transport equation, which is defined by the solid transport discharge. In Section 2.2 we present some experimental formulae that can be considered.

Shallow Water system and the sediment transport equation form a coupled system that is described in Section 2.6.

\subsection{Hydrodynamical model: Shallow Water equations}

We consider a one-dimensional channel with variable bottom and constant rectangular section. If the bottom is fixed, we have the equations

$$
\left\{\begin{array}{l}
\frac{\partial h}{\partial t}+\frac{\partial q}{\partial x}=0 \\
\frac{\partial q^{2}}{\partial t}+\frac{\partial}{\partial x}\left(\frac{q}{h}+\frac{1}{2} g h^{2}\right)=g h \frac{d H}{d x}-g h S_{f} .
\end{array}\right.
$$

Where by $x$ we denote the horizontal variable at the axis of the channel and $t$ is the time variable. By $q(x, t)$ and $h(x, t)$ we denote the discharge and the height of the water column respectively. $g$ is the gravity constant and $H(x)$ is the bathymetry function respect to a fixed reference level $\left(A_{R}\right)$. (See Figure 2(a)).

The term $S_{f}$ models the friction term, for Manning law we have

$$
S_{f}=\frac{g \eta^{2}|u| u}{R_{h}^{4 / 3}},
$$

where $\eta$ is the Manning's coefficient, $R_{h}$ is the hydraulic ratio, which can be approximated by $h$ and $u$ is the velocity of the fluid $\left(u(x, t)=\frac{q(x, t)}{h(x, t)}\right)$.

To study the problem of sediment transport it is necessary to consider a sediment layer, $z_{b}$ and a fixed bottom (without sediment), $z_{f}=-H+A_{R}\left(z_{f}+H=A_{R}\right)$. In this case the system of equations (2) can be written as follows (See Figure 2(b)),

$$
\left\{\begin{array}{l}
\frac{\partial h}{\partial t}+\frac{\partial q}{\partial x}=0 \\
\frac{\partial q}{\partial t}+\frac{\partial}{\partial x}\left(\frac{q^{2}}{h}+\frac{1}{2} g h^{2}\right)=-g h \frac{\partial z_{b}}{\partial x}+g h \frac{d H}{d x}-g h S_{f} .
\end{array}\right.
$$




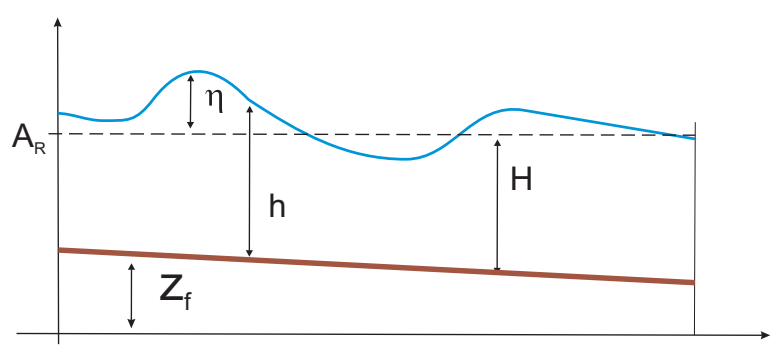

(a) Without sediment.

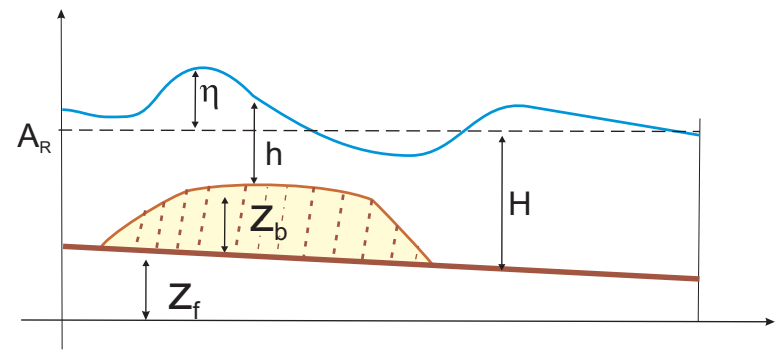

(b) A fixed bottom and a sediment layer.

Figure 2: Possible configurations of the bottom of the channel in function of the presence or not of a sediment layer

\subsection{Morphodynamical model}

The equation that describes sediment transport is a continuity equation and the expression of the conservation sediment volume equation is given by

$$
\frac{\partial z_{b}}{\partial t}+\xi \frac{\partial q_{b}}{\partial x}=0
$$

where $\xi=1 /\left(1-\rho_{0}\right)$ and $\rho_{0}$ is the porosity of the sediment layer. By $q_{b}=q_{b}(h, q)$ we denote the solid transport discharge.

\subsection{Solid transport discharge}

In this section we present the most popular formulae to define the solid transport discharge, $q_{b}$. The study of the definition of the solid transport discharge can be seen as a deterministic problem or a probabilistic one. For example, deterministic methods have been proposed by Duboy and MeyerPeter\& Müller ([17], [41]); and probabilistic methods by Kalinske ([34], [35]) and Einstein ([18], [19]). Different estimations of the solid transport discharge $q_{b}$ have been obtained by empirical methods.

In what follows, we present some formulae for $q_{b}$ corresponding to deterministic models. They were proposed for granular and non-cohesive sediments. They were obtained for stationary flux in rivers. Nevertheless, it can also be applied to fluids in tides, by the effects of the waves and the currents, because the time response of the sediment is very small in comparison with the period of the tide or the wave. These formulae do not include any gradient pressure effect to model grain falling when the velocity of the fluid is zero.

All models considered in this work, except Grass model, have into account that the movement of the granular sediment begins when the shear stress $(\tau)$ is bigger than a certain critical shear stress $\left(\tau_{c}\right)$.

Usually, shear stress can be written in terms of the hydrodynamical unknowns, $h$ and $q$, using the friction term

$$
\tau=\gamma R_{h}\left|S_{f}\right|,
$$

where $\gamma$ denotes the specific weight of the fluid $\gamma=g \rho$, being $\rho$ the water density and $S_{f}$ is defined by (3). Usually, Manning's coefficient $\eta$ over the sediment layer is not equal to Manning's coefficient over the fixed bottom.

Nevertheless, the shear stress is usually presented in a non-dimensional form in $q_{b}$. If we denote by $\tau_{*}$ and $\tau_{* c}$ the non-dimensional shear stress and the critical shear stress, respectively, then

$$
\tau_{*}=\frac{\tau}{\left(\gamma_{s}-\gamma\right) d}, \quad \tau_{* c}=\frac{\tau}{\left(\gamma_{s}-\gamma\right) d} .
$$


Where $\gamma_{s}$ denotes the specific sediment weight $\gamma_{s}=g \rho_{s}$, being $\rho_{s}$ the sediment density, and $d$ denotes the sediment grain size (diameter).

Using (6) and (7), $\tau_{*}$ can be written in function of the relative density of fluids $G=\rho_{s} / \rho$.

$$
\tau_{*}=\frac{g \eta^{2} u^{2}}{(G-1) d R_{h}^{1 / 3}} .
$$

To determine $\tau_{* c}$ many experiments have been performed in different works. Concretely, Shields proposed the well-known Shields-diagram. He did the measurement for bigger diameters and after extrapolate.

Some of the most usual formulae for rivers are:

- Grass equation:

Grass ([25]) proposed the following formula for the solid transport discharge,

$$
q_{b}=A_{g} \frac{q}{h}\left|\frac{q}{h}\right|^{m_{g}-1}, 1 \leqslant m_{g} \leqslant 4,
$$

where the constant $A_{g}\left(s^{2} / m\right)$ is usually obtained by experimental data and takes into account the grain diameter and the cinematic viscosity. The usual value of the exponent $m_{g}$ is set to $m_{g}=3$, obtaining

$$
q_{b}=A_{g} u^{3} .
$$

The coefficient $A_{g}$ takes values between 0 and 1 . When it is closed to 0 the model reflects a weak interaction between the sediment and the fluid.

Note that one of the main characteristics of this model is that the critical shear stress is set to zero, so the sediment movement begins at the same instant that beginning fluid motion.

- Meyer-Peter\&Müller's equation:

Meyer-Peter\& Müller [41] (MP\&M in what follows) developed one of the most known formulae for the solid transport discharge, based on median grain diameter $d_{50}$ (see [55]). Later, Chien [14] proved that original formula can be reduced to the following expression

$$
\frac{q_{b}}{\sqrt{(G-1) g d_{i}^{3}}}=\operatorname{sgn}(u) 8\left(\tau_{*}-\tau_{* c}\right)^{3 / 2},
$$

where $\tau_{* c}$ usually is set to 0.047 .

This expression is one of the most used and it is applied to rivers and channels with slope lower than $2 \%$. A more complex formula for non rectangular cross-section channels can be found in [52] and [49].

- Fernández Luque\&Van Beek equation (FL\&V):

This formula is very similar to (10), and in fact, only one coefficient varies,

$$
\frac{q_{b}}{\sqrt{(G-1) g d_{i}^{3}}}=\operatorname{sgn}(u) 5.7\left(\tau_{*}-\tau_{* c}\right)^{1 / 3} .
$$

- Van Rijn's equation:

Van Rijn ([58]-[60]) developed the following formula for the solid transport discharge,

$$
\frac{q_{b}}{\sqrt{(G-1) g d_{i}^{3}}}=\frac{0.005}{C_{D}^{1.7}}\left(\frac{d}{h}\right)^{0.2} \tau_{*}^{1 / 2}\left(\tau_{*}^{1 / 2}-\tau_{* c}^{1 / 2}\right)^{2.4}
$$


where $C_{D}$ is the dragging coefficient. Another formula based on a complex theory mixing empirical results and fundamental physics was proposed by Van Rijn (see [55], for example).

- Nielsen formula:

Nielsen ([42]) developed the following formula

$$
\frac{q_{b}}{\sqrt{(G-1) g d_{i}^{3}}}=\operatorname{sgn}(u) 12 \sqrt{\tau_{*}}\left(\tau_{*}-\tau_{* c}\right) .
$$

In this case the usual value of $\tau_{* c}$ is set equal to $\tau_{* c}=0.05$.

All these formulae have a range of application in function of grain size, the slope of the bottom, the Froude number and the relative density $G$. For example in the case of the MP\&M it is applied for a $0.4 \leq d \leq 29 \mathrm{~mm}$, slopes of the bottom smaller than 0.02 and $1.25 \leq G \leq 4.2$ (see [45]). In Section 6.3 we have used MP\&M model in order to compare numerical results with experimental data.

\subsection{Unified formulation for solid transport discharge formulae}

The different formulae of $q_{b}$ shown in the previous subsection can be written under a same structure as follows:

$$
q_{b}(h, q)=c_{1} g_{2}(h, q)\left(c_{2}+c_{3} g_{1}(h, q)\right)^{m},
$$

where $m, c_{1}, c_{2}$ and $c_{3}$ are constant values and $g_{1}, g_{2}$ are scalar functions of $h$ and $q$. For example,

- Grass model corresponds to

$$
c_{1}=A_{g}, g_{2}(h, q)=u, c_{2}=0, c_{1}=1, g_{1}(h, q)=|u|, m=m_{g}-1 .
$$

- Meyer-Peter\&Müller's model corresponds to,

$$
\begin{array}{cc}
c_{1}=8 \sqrt{(G-1) g d_{i}^{3}}, \quad c_{2}=-0.047, \quad c_{3}=\frac{\gamma \eta^{2}}{\left(\gamma_{s}-\gamma\right) d_{i}}, \\
g_{1}(q, h)=\frac{q^{2}}{h^{2} R_{h}^{1 / 3}}, \quad g_{2}(q, h)=\operatorname{sgn}(u), \quad m=1.5 .
\end{array}
$$

\subsection{Comparison of the different models with Grass model.}

We observe that if we set $\tau_{* c}=0$ at the previous models, then it is possible to interpret them as particular cases of Grass model, for different definitions of $A_{g}$ and $m_{g}$. As the value of $A_{g}$ represents the type of interaction between fluid and sediment, we can look for it in the different models.

We analyze in detail the MP\&M's model for $\tau_{* c}=0$. The solid transport discharge in this case can be written under the structure (9), where

$$
A_{g}=\frac{8 \sqrt{g} \gamma \eta^{3}}{\left(\gamma_{s}-\gamma\right) R_{h}^{1 / 2}}
$$

We observe that $A_{g}$ cannot be constant because it depends on the hydraulic ratio. $A_{g}$ depends also on the specific sediment weight, the specific water weight and Manning's coefficient. Nevertheless, for $\tau_{* c}=0$, the MP\&M's model does not depend on grain diameter $d_{i}$. In fact, this is similar for almost all the considered models except for Van Rijn's model.

We consider the following values $\gamma=g \cdot 1000, \gamma_{s}=g \cdot 2600, \eta=0.0196$. With this values, we obtain 


$$
A_{g} \approx \frac{1.17915995565 \cdot 10^{-5}}{R_{h}^{1 / 2}}
$$

If the hydraulic ratio $R_{h}$ is approximated by $h$, and if $h \approx 10 \mathrm{~cm}$, we obtain $A_{g} \approx 1.17915995565$. $10^{-9 / 2}$. For values of $h \approx 1 \mathrm{~cm}$ we obtain $A_{g} \approx 1.17915995565 \cdot 10^{-4}$. In any case, we observe that MP\&M always models a weak interaction between fluid and sediment.

As shown in Table 1, all models considered here correspond to a weak interaction between fluid and sediment. In fact, this is one of the main problems in order to obtain precise numerical approximations. As the interaction between the fluid and the sediment is very weak, the velocity of the fluid is much greater than the velocity of the sediment. Consequently, low order numerical methods do not capture well the sediment evolution; they are usually very diffusive (see test in Section 6.1). So, it is necessary to use high order numerical schemes in order to avoid this phenomena.

\begin{tabular}{|c|c|c|c|}
\hline Formula & $A_{g}$ & $m_{g}$ & Approximation \\
\hline \hline MP\&M & $A_{g}=\frac{8 g^{2} \gamma \eta^{3}}{\left(\gamma_{s}-\gamma\right) R_{h}^{1 / 2}}$ & 3 & $3.62306 \times 10^{-3} \frac{1}{R_{h}^{1 / 2}}$ \\
\hline \multirow{2}{*}{ Nielsen } & $A_{g}=\frac{12 g^{2} \gamma \eta^{3}}{\left(\gamma_{s}-\gamma\right) R_{h}^{1 / 2}}$ & 3 & $5.43459 \times 10^{-3} \frac{1}{R_{h}^{1 / 2}}$ \\
\hline Van Rijn & $A_{g}=\frac{0.005 g^{2.2} \eta^{3.4}}{(G-1)^{1.2} h^{0.2} R_{h}^{1.7 / 3} C_{D}^{1.7}}$ & 3.4 & $0.6751 \times 10^{-6} \frac{1}{C_{D}^{1.7} R_{h}^{1.7 / 3}}$ \\
\hline FL\&V & $A_{g}=\frac{5.7 g^{2} \gamma \eta^{3}}{\left(\gamma_{s}-\gamma\right) R_{h}^{1 / 2}}$ & 3 & $2.58143 \times 10^{-3} \frac{1}{R_{h}^{1 / 2}}$ \\
\hline
\end{tabular}

Table 1: Comparison with Grass model.

\subsection{Coupled model}

The system of equations used in this work is obtained by coupling shallow water equations (4) and the sediment transport equation (5). The expression of this system is as follows

$$
\left\{\begin{array}{l}
\frac{\partial h}{\partial t}+\frac{\partial q}{\partial x}=0 \\
\frac{\partial q}{\partial t}+\frac{\partial}{\partial x}\left(\frac{q^{2}}{h}+\frac{1}{2} g h^{2}\right)=-g h \frac{\partial z_{b}}{\partial x}+g h \frac{d H}{d x}-g h S_{f}, \\
\frac{\partial z_{b}}{\partial x}+\xi \frac{\partial}{\partial x} q_{b}(h, q)=0,
\end{array}\right.
$$

where $S_{f}$ is the friction term modeling the drag effects between the fluid and the bed defined by (3). Note that, if the variable $S=H-z_{b}$ is defined, as $\frac{\partial S}{\partial t}=-\frac{\partial z_{b}}{\partial t}$, (15) could be rewritten as 


$$
\left\{\begin{array}{l}
\frac{\partial h}{\partial t}+\frac{\partial q}{\partial x}=0 \\
\frac{\partial q}{\partial t}+\frac{\partial}{\partial x}\left(\frac{q^{2}}{h}+\frac{1}{2} g h^{2}\right)=g h \frac{\partial S}{\partial x}-g h S_{f} \\
\frac{\partial S}{\partial t}-\xi \frac{\partial q_{b}}{\partial x}=0
\end{array}\right.
$$

Note that, system (16) can be written as an hyperbolic system with a non-conservative term and a source term $S_{F}$

$$
\frac{\partial W}{\partial t}+\frac{\partial F(W)}{\partial x}=B(W) \frac{\partial W}{\partial x}+S_{F}
$$

where

$$
W=\left[\begin{array}{c}
h \\
q \\
S
\end{array}\right], \quad F=\left[\begin{array}{c}
q \\
\frac{q^{2}}{h}+\frac{1}{2} g h^{2} \\
-\xi q_{b}
\end{array}\right], \quad B(W)=\left[\begin{array}{ccc}
0 & 0 & 0 \\
0 & 0 & g h \\
0 & 0 & 0
\end{array}\right], \quad S_{F}=\left[\begin{array}{c}
0 \\
-g h S_{f} \\
0
\end{array}\right]
$$

Finally, system (17) can be written as a non-conservative hyperbolic system

$$
\frac{\partial W}{\partial t}+\mathcal{A}(W) \frac{\partial W}{\partial x}=S_{f}, x \in \mathbb{R}, t>0,
$$

where

$$
\mathcal{A}(W)=A(W)-B(W),
$$

and $A(W)$ is the Jacobian matrix of $F(W)$,

$$
A(W)=\frac{\partial F}{\partial W}(W)=\left[\begin{array}{ccc}
0 & 1 & 0 \\
-\frac{q^{2}}{h^{2}}+g h & 2 \frac{q}{h} & 0 \\
-\xi \frac{\partial q_{b}}{\partial h} & -\xi \frac{\partial q_{b}}{\partial q} & 0
\end{array}\right] .
$$

Then, by the definitions of $A(W)$ and $B(W)$ we obtain that

$$
\mathcal{A}(W)=\left[\begin{array}{ccc}
0 & 1 & 0 \\
-\frac{q^{2}}{h^{2}}+g h & 2 \frac{q}{h} & -g h \\
-\xi \frac{\partial q_{b}}{\partial h} & -\xi \frac{\partial q_{b}}{\partial q} & 0
\end{array}\right] .
$$

The eigenvalues of the matrix $\mathcal{A}(W)$ can be obtained as roots of the characteristic polynomial

$$
P(\lambda)=\lambda^{3}+a_{1} \lambda^{2}+a_{2} \lambda+a_{3},
$$

where

$$
a_{1}=-2 \frac{q}{h}, \quad a_{2}=\frac{q^{2}}{h^{2}}-g h\left(1+\xi \frac{\partial q_{b}}{\partial q}\right) \quad \text { and } a_{3}=-g h \xi \frac{\partial q_{b}}{\partial h} .
$$

The roots of this polynomial are 


$$
\begin{aligned}
& \lambda_{1}=2 \sqrt{-Q} \cos (\theta / 3)-a_{1} / 3, \\
& \lambda_{2}=2 \sqrt{-Q} \cos ((\theta+2 \pi) / 3)-a_{1} / 3, \\
& \lambda_{3}=2 \sqrt{-Q} \cos ((\theta+4 \pi) / 3)-a_{1} / 3,
\end{aligned}
$$

where $Q=\left(3 a_{2}-a_{1}^{2}\right) / 9, \theta=\arccos \left(R / \sqrt{-Q^{3}}\right)$ and $R=\left(9 a_{1} a_{2}-27 a_{3}+2 a_{1}^{3}\right) / 54$. The eigenvalues are real if $Q^{3}+R^{2}<0$. In the case of Grass model, it can be proven that all eigenvalues are real. Although in general we cannot prove it for the other considered models, we have not found any complex eigenvalue in our numerical tests.

The associated eigenvectors are

$$
R_{j}=\left[\begin{array}{c}
1 \\
\lambda_{k} \\
\frac{\left(\frac{q}{h}-\lambda_{k}\right)^{2}}{g h}-1
\end{array}\right], \quad j=1,2,3 .
$$

We note that for a fixed bottom $\left(q_{b}=0\right)$ the eigenvalues are

$$
\frac{q}{h}+\sqrt{g h}, \quad \frac{q}{h}-\sqrt{g h} \quad \text { and } 0 .
$$

By simplicity we will suppose that the system is strictly hyperbolic, where $\lambda_{i} \neq \lambda_{j}$, if $i \neq j$ and all of them are real.

Moreover, we must remark that the non-conservative product $\mathcal{A}(W) \cdot W_{x}$, has not sense as a distribution when $W$ presents a discontinuity. Following the theory developed by G. Dal Maso, P. G. LeFloch and F. Murat ([16]), can be defined in a rigorous way the non-conservative product, introducing a family of paths in $\Omega$.

Definition $1 A$ family of paths in $\Omega \subset \mathbb{R}^{N}$ is a local-Lipschitz application

$$
\Phi:[0,1] \times \Omega \times \Omega \longrightarrow \Omega,
$$

that satisfies the following properties:

1. $\Phi\left(0 ; W_{L}, W_{R}\right)=W_{L}$ and $\Phi\left(1 ; W_{L}, W_{R}\right)=W_{R}$, for all $W_{L}, W_{R} \in \Omega$.

2. for a bounded set $\mathcal{O} \subset \Omega$, there exists a constant $k$ such that

$$
\left|\frac{\partial \Phi}{\partial s}\left(s ; W_{L}, W_{R}\right)\right| \leqslant k\left|W_{L}-W_{R}\right|,
$$

for all $W_{L}, W_{R} \in \mathcal{O}$ and for almost all $s \in[0,1]$.

3. for a given bounded set $\mathcal{O} \subset \Omega$, there exists a constant $K$ such that,

$$
\left|\frac{\partial \Phi}{\partial s}\left(s ; W_{L}^{1}, W_{R}^{1}\right)-\frac{\partial \Phi}{\partial s}\left(s ; W_{L}^{2}, W_{R}^{2}\right)\right| \leqslant K\left(\left|W_{L}^{1}-W_{L}^{2}\right|+\left|W_{R}^{1}-W_{R}^{2}\right|\right),
$$

for all $W_{L}^{1}, W_{R}^{1}, W_{L}^{2}, W_{R}^{1} \in \mathcal{O}$ and for almost all $s \in[0,1]$.

Once a family of paths is chosen, $\Phi$ in $\Omega$, given a function $W \in\left(L^{\infty}\left(\mathbb{R} \times \mathbb{R}^{+}\right) \cap B V\left(\mathbb{R} \times \mathbb{R}^{+}\right)\right)^{N}$ it is possible to give a sense to the non-conservative product as a Borel measure (see [43]), which is denoted by $\left[\mathcal{A}(W) W_{x}\right]_{\Phi}$ and weak solutions of (19) are the functions satisfying the equality 


$$
W_{t}+\left[\mathcal{A}(W) W_{x}\right]_{\Phi}=S_{f} .
$$

The choice of the family of paths is very important, because it determines the propagation speed of shocks. Nevertheless, in general, it is very difficult to parametrice the optimal choice, that must be related to the physics of the problem. In practice, the simplest choice is given by a family of segments,

$$
\Phi\left(s ; W_{L}, W_{R}\right)=W_{L}+s\left(W_{R}-W_{L}\right), s \in[0,1],
$$

which corresponds to the non-conservative product definition proposed by Volpert [63].

\section{Finite volume method for non conservative hyperbolic sys- tems}

Friction term $S_{f}$ will be discretized in an semi-implicit way (see [8]), so, in what follows we consider the system

$$
\frac{\partial W}{\partial t}+\mathcal{A}(W) \frac{\partial W}{\partial x}=0, x \in \mathbb{R}, t>0 .
$$

We consider a partition of the interval $[0, L]$ in cells defined by $I_{i}=\left[x_{i-1 / 2}, x_{i+1 / 2}\right], i \in \mathbb{Z}$. For the sake of simplicity, we suppose that all of them have the same length $\Delta x$ and $x_{i+\frac{1}{2}}=i \Delta x$. $x_{i}=(i-1 / 2) \Delta x$ is the center of the control volume $I_{i}$. Let $\Delta t$ be the time step and $t^{n}=n \Delta t$.

Then we denote by $W_{i}^{n}$ an approximation on the mean value of $W$ over $I_{i}$ at time $t=t^{n}$,

$$
W_{i}^{n} \cong \frac{1}{\Delta x} \int_{x_{i-1 / 2}}^{x_{i+1 / 2}} W\left(x, t^{n}\right) d x .
$$

To approximate $W_{i}^{n}$ we consider two different types of schemes, the generalized Roe method for nonconservative hyperbolic systems and schemes with flux limiters, which are of second order in regular areas, at least for linear problems. In the case of a non-conservative system it is also necessary to define a generalization of Roe matrix. The definition of Roe matrix is also associated to the choice of a family of paths.

In [57] the following definition is introduced.

Definition 2 For a given family of paths $\Psi$, a function $\mathcal{A}: \Omega \times \Omega \longrightarrow \mathcal{M}_{N}(\mathbb{R})$ is a Roe linearization of system (25), if it satisfies the following properties:

1. For each $W_{L}, W_{R} \in \Omega, \mathcal{A}_{\Psi}\left(W_{L}, W_{R}\right)$ has $N$ real and different eigenvalues.

2. $\mathcal{A}_{\Psi}(W, W)=\mathcal{A}(W)$, for all $W \in \Omega$.

3. for each $W_{L}, W_{R} \in \Omega$,

$$
\mathcal{A}_{\Psi}\left(W_{L}, W_{R}\right)\left(W_{R}-W_{L}\right)=\int_{0}^{1} \mathcal{A}\left[\Psi\left(s ; W_{L}, W_{R}\right)\right] \frac{\partial \Psi}{\partial s}\left(s ; W_{L}, W_{R}\right) d s .
$$

We observe that if $\mathcal{A}(W)$ is the Jacobian matrix of a regular flux $F(W),(26)$ is independent from the family of paths and it is reduced to the usual Roe property

$$
\mathcal{A}_{\Psi}\left(W_{L}, W_{R}\right)\left(W_{R}-W_{L}\right)=F\left(W_{R}\right)-F\left(W_{L}\right) .
$$

We will use the following notation: we denote

$$
\mathcal{A}_{i+1 / 2}=\mathcal{A}_{\Psi}\left(W_{i}^{n}, W_{i+1}^{n}\right)
$$


the Roe matrix associated to the states $W_{i}$ and $W_{i+1}$, with eigenvalues

$$
\lambda_{1}^{i+1 / 2}<\lambda_{2}^{i+1 / 2}<\ldots<\lambda_{N}^{i+1 / 2}
$$

and $\left\{R_{l}^{i+1 / 2}\right\}_{l=1}^{N}$ is the base of the associated eigenvectors. By $\mathcal{K}_{i+1 / 2}$ we denote the $N \times N$ matrix whose columns are eigenvectors and by $\mathcal{L}_{i+1 / 2}$, the diagonal matrix of eigenvalues. We will also use the following matrices $\mathcal{L}_{i+1 / 2}^{+}, \mathcal{L}_{i+1 / 2}^{-}, \mathcal{A}_{i+1 / 2}^{+}, \mathcal{A}_{i+1 / 2}^{-}$and $\left|\mathcal{A}_{i+1 / 2}^{+}\right|$:

$$
\begin{aligned}
\mathcal{L}_{i+1 / 2}^{ \pm} & =\left[\begin{array}{ccc}
\left(\lambda_{1}^{i+1 / 2}\right)^{ \pm} & 0 & 0 \\
0 & \left(\lambda_{2}^{i+1 / 2}\right)^{ \pm} & 0 \\
0 & 0 & \left(\lambda_{3}^{i+1 / 2}\right)^{ \pm}
\end{array}\right], \quad\left(\lambda_{k}^{i+1 / 2}\right)^{ \pm}=\frac{\lambda_{k}^{i+1 / 2} \pm\left|\lambda_{k}^{i+1 / 2}\right|}{2}, \\
\mathcal{A}_{i+1 / 2}^{ \pm} & =\mathcal{K}_{i+1 / 2} \mathcal{L}_{i+1 / 2}^{ \pm} \mathcal{K}_{i+1 / 2}^{-1},
\end{aligned}
$$

and

$$
\left|\mathcal{A}_{i+1 / 2}\right|=\mathcal{A}_{i+1 / 2}^{+}-\mathcal{A}_{i+1 / 2}^{-} .
$$

The numerical scheme progresses in time as follows: once the approximations at time $t^{n}, W_{i}^{n}$, have been calculated, a Linear Riemann problem is considered at each intercell $x=x_{i+1 / 2}$ with the associated Roe matrix $\mathcal{A}_{i+1 / 2}$ and the constant states $W_{i}^{n}$ and $W_{i}^{n+1}$, respectively. The approximations at time $t^{n+1}, W_{i}^{n+1}$, are obtained by averaging in the cells the solutions of the Riemann problem. As in the case of a system of conservation laws, some calculations allow to show that, under the hypothesis,

$$
x_{i-1 / 2}+\lambda_{1}^{i-1 / 2} \Delta t \leq x_{i} \leq x_{i+1 / 2}+\lambda_{N}^{i+1 / 2} \Delta t,
$$

the approximations at time $t^{n+1}$ can be obtained by the formula:

$$
W_{i}^{n+1}=W_{i}^{n}-\frac{\Delta t}{\Delta x}\left(\mathcal{A}_{\mathcal{D}, i-1 / 2}^{+}\left(W_{i}^{n}-W_{i-1}^{n}\right)+\mathcal{A}_{\mathcal{D}, i+1 / 2}^{-}\left(W_{i+1}^{n}-W_{i}^{n}\right)\right),
$$

where,

$$
\mathcal{A}_{\mathcal{D}, i+1 / 2}^{ \pm}=\frac{\mathcal{A}_{i+1 / 2} \pm \mathcal{D}_{i+1 / 2}}{2} .
$$

$\mathcal{D}_{i+1 / 2}$ is the viscosity matrix of the numerical scheme defined by:

a) Roe method,

$$
\mathcal{D}_{i+1 / 2}=\left|\mathcal{A}_{i+1 / 2}\right|
$$

b) Method with flux limiters: the idea is to use a combination of Roe method (which is only of order one) near discontinuities, and the generalization of Lax-Wendroff method (which is second order in space and time, at least for linear systems) in regular areas. The obtained scheme corresponds to define

$$
\mathcal{D}_{i+1 / 2}=\left|\mathcal{A}_{i+1 / 2}\right|-\mathcal{K}_{i+1 / 2}\left(\mathcal{L}_{\varphi}\right)_{i+1 / 2} \mathcal{K}_{i+1 / 2}^{-1}
$$

with,

$$
\mathcal{L}_{\varphi}=\operatorname{Diag}\left\{\left(\operatorname{sgn}\left(\lambda_{j}\right)-\frac{\Delta t}{\Delta x} \lambda_{j}\right) \lambda_{j} \varphi\left(r_{j}\right), j=1, \ldots, N .\right\} .
$$

The function $\varphi$ is the flux-limiter and 


$$
r_{j}=\frac{\alpha_{j, i_{0}}}{\alpha_{j, i}}, \quad \alpha_{j, i}=\left[\mathcal{K}^{-1}\left(W_{i+1}-W_{i}\right)\right]_{j}, \quad \text { with } i_{0}=i-\operatorname{sgn}\left(\lambda_{j}\right)
$$

Some classical examples of flux limiters function are (see [21] and [39]):

- Van Leer flux limiter:

$$
\varphi(r)=\frac{|r|+r}{1+|r|}
$$

- The family of flux limiters functions defined by

$$
\varphi(r)=\max (0, \min (1, \beta r), \min (\beta, r)) .
$$

If $\beta=1$ we have the minmod limiter, $\beta=2$ corresponds to superbee limiter.

Moreover it can be proven that scheme (30) is well-balanced.

Remark 1 In practice, to ensure (29) we use the CFL condition

$$
\max \left\{\left|\lambda_{l}^{i+1 / 2}\right|, 1 \leq l \leq N, i \in \mathbb{Z}\right\} \frac{\Delta t}{\Delta x} \leq \gamma,
$$

with $0<\gamma \leq 1$.

Taking into account that the system (17) has a conservative term and a non conservative product, the numerical scheme could be rewritten as follows:

$$
\begin{aligned}
W_{i}^{n+1}=W_{i}^{n} & +\left(G_{i+1 / 2}-G_{i-1 / 2}\right) \\
& +\frac{\Delta t}{2 \Delta x}\left(B_{i-1 / 2}\left(W_{i}^{n}-W_{i-1}^{n}\right)+B_{i+1 / 2}\left(W_{i+1}^{n}-W_{i}^{n}\right)\right)
\end{aligned}
$$

where

$$
G_{i+1 / 2}=\frac{1}{2}\left(F\left(W_{i}^{n}\right)+F\left(W_{i+1}^{n}\right)\right)-\frac{1}{2} \mathcal{D}_{i+1 / 2}\left(W_{i+1}^{n}-W_{i}^{n}\right) .
$$

\section{High order finite volume scheme based on state reconstruc- tions}

There exist different techniques to obtain high order methods. A possibility is to use state reconstructions. Firstly a state reconstruction operator $P^{t}$ is considered, that is, an operator that associates, to a given sequence $\left\{W_{i}(t)\right\}$, two new sequences $\left\{W_{i+1 / 2}^{-}(t)\right\},\left\{W_{i+1 / 2}^{+}(t)\right\}$ in such a way that, whenever

$$
W_{i}(t)=\frac{1}{\Delta x} \int_{I_{i}} W(x, t) d x, \forall i \in \mathbb{Z}
$$

for some regular function $W$, then

$$
W_{i+1 / 2}^{ \pm}(t)=W\left(x_{i+1 / 2}, t\right)+O\left(\Delta x^{p}\right), \forall i \in \mathbb{Z} .
$$

For conservative hyperbolic systems it is enough with these reconstructions, nevertheless for nonconservative terms it is necessary to know the state reconstruction over the interval $I_{i}$. That happens because if $\mathcal{A}(W) W_{x}$ has an associated flux and we integrate it over the control volume, then only the states at the boundary of $I_{i}\left(x_{i-1 / 2}\right.$ and $\left.x_{i+1 / 2}\right)$ are necessary. However if an associated flux does not exist, we must integrate $\mathcal{A}(W) W_{x}$ over all the interval. 
Then, firstly, over each control volume $I_{i}$, at each instant $t>0$, we define a regular function $P_{i}^{t}$ such that

$$
\lim _{x \rightarrow x_{i-1 / 2}^{+}} P_{i}^{t}(x)=W_{i-1 / 2}^{+}(t), \quad \lim _{x \rightarrow x_{i+1 / 2}^{-}} P_{i}^{t}(x)=W_{i+1 / 2}^{-}(t) .
$$

In [10] the following numerical scheme is proposed,

$$
\begin{aligned}
W_{i}^{\prime}=-\frac{1}{\Delta x}\left(\mathcal{A}_{i-1 / 2}^{+}\left(W_{i-1 / 2}^{+}(t)-W_{i-1 / 2}^{-}(t)\right)\right. & +\mathcal{A}_{i+1 / 2}^{-}\left(W_{i+1 / 2}^{+}(t)-W_{i+1 / 2}^{-}(t)\right) \\
& \left.+\int_{x_{i-1 / 2}}^{x_{i+1 / 2}} \mathcal{A}\left[P_{i}^{t}(x)\right] \frac{d}{d x} P_{i}^{t}(x) d x\right),
\end{aligned}
$$

where $P_{i}^{t}$ is a regular function that verifies (38). Applied to our system (17) with a conservative part and a non-conservative product, it can be rewritten as

$$
\begin{aligned}
W_{i}^{\prime} & =\frac{\Delta t}{\Delta x}\left(\widetilde{G}_{i-1 / 2}-\widetilde{G}_{i+1 / 2}\right) \\
& +\frac{\Delta t}{2 \Delta x}\left(B_{i-1 / 2} \cdot\left(W_{i-1 / 2}^{+}-W_{i-1 / 2}^{-}\right)+B_{i+1 / 2} \cdot\left(W_{i+1 / 2}^{+}-W_{i+1 / 2}^{-}\right)\right) \\
& +\frac{\Delta t}{\Delta x} \mathcal{I}_{B, i},
\end{aligned}
$$

where,

$$
\widetilde{G}_{i-1 / 2}=\frac{1}{2}\left(F\left(W_{i+1 / 2}^{-}\right)+F\left(W_{i+1 / 2}^{+}\right)\right)-\frac{1}{2}\left|\mathcal{A}_{i+1 / 2}\right| \cdot\left(W_{i+1 / 2}^{+}-W_{i+1 / 2}^{-}\right) .
$$

$\mathcal{A}_{i+1 / 2}$ is the Roe matrix and

$$
\mathcal{I}_{B, i}=\int_{x_{i-1 / 2}}^{x_{i+1 / 2}} B\left[\widetilde{P}_{i}^{t}\right] \frac{d}{d x} P_{i}^{t}(x) d x .
$$

Effectively, we see that the main difference between the conservative and the non-conservative system is that the conservative one is independent of the definition of $P_{i}^{t}$, only depends on $W_{i \pm 1 / 2}^{ \pm}$. The consequences are that the order of the numerical scheme depends upon the order of the state reconstruction operator $P_{i}^{t}$ and its derivative. Concretely, in [10] the following theorem is proven.

THEOREM 1 If $\mathcal{A}$ is order $\mathcal{C}^{2}$ with bounded derivatives and $\mathcal{A}_{\Psi}$ is bounded. We suppose that the state reconstruction operator of order $p$ verifies that for a sequence defined by

$$
W_{i}=\frac{1}{\Delta x} \int_{I_{i}} W(x) d x
$$

for some regular function $W$,

$$
\begin{array}{ll}
P_{i}\left(x ; W_{i-l}, \ldots, W_{i+r}\right)=W(x)+O\left(\Delta x^{q}\right), & \forall x \in I_{i}, \\
\frac{d}{d x} P_{i}(x)=W^{\prime}+O\left(\Delta x^{r}\right), & \forall x \in I_{i} .
\end{array}
$$

Then (39) provides an approximation of at least order $\alpha=\min (p, q+1, r+1)$ for system (25) in the following sense:

$$
\begin{aligned}
& \left(\mathcal{A}_{i-1 / 2}^{+}\left(W_{i-1 / 2}^{+}(t)-W_{i-1 / 2}^{-}(t)\right)+\mathcal{A}_{i+1 / 2}^{-}\left(W_{i+1 / 2}^{+}(t)-W_{i+1 / 2}^{-}(t)\right)\right. \\
& \left.+\int_{x_{i-1 / 2}}^{x_{i+1 / 2}} \mathcal{A}\left[P_{i}^{t}(x)\right] \frac{d}{d x} P_{i}^{t}(x) d x\right)=\int_{x_{i-1 / 2}}^{x_{i+1 / 2}} \mathcal{A}(W(x, t)) W_{x}(x, t) d x+O\left(\Delta x^{\alpha}\right),
\end{aligned}
$$

for any sufficiently smooth solution $W$. 
Remark 2 For usual state reconstruction operators, we have $r \leq q \leq p$ and then, the order of (39) is $\gamma=r+1$ for non-conservative systems, by while for conservative systems the order is $p$. So, the order of the numerical scheme for non-conservative systems is usually smaller than for conservative one.

In this paper we have used state operator reconstruction defined by WENO reconstructions with stencils of $r$ points. So, we denote by r-WENO method the scheme obtained by this state reconstruction. For this operator of reconstruction we obtain at the internal points of the control volume only order $r$ and $q=r-1$ for their derivatives, so, by previous theorem, the scheme is order $r$.

For time evolution, we have used the high order TVD Runge-Kutta method proposed in [54].

For the numerical tests presented in Section 6 we use a 3-point Gauss quadrature formula to approximate integral (42).

\section{Roe Matrix and numerical treatment of transitions close the critical shear stress}

In this section we study the problem of determining Roe matrices for the coupled model formed by shallow water equations and sediment transport defined by the family of solid transport discharge (defined in Section 2.3). In the particular case of Grass model it is possible to determine a Roe matrix, when the family of paths is defined by segments. Nevertheless the calculus of the Roe matrices is complicated for the other models presented in this work, and its implementation is very costly. So, in practice we use an approximation of Roe matrix. In subsection 5.3 we present the numerical treatment that we perform in transition interfaces with-without sediment, with-without movement of the sediment layer.

\subsection{Roe Matrix for Grass model}

In the particular case where Grass model is considered and the family of paths is defined by segments (24), Roe matrix is (See [31]),

$$
\mathcal{A}_{\Psi}\left(W_{L}, W_{R}\right)=\left[\begin{array}{ccc}
0 & 1 & 0 \\
-(\widetilde{u})^{2}+(\widetilde{c})^{2} & 2 \widetilde{u} & (\widetilde{c})^{2} \\
-\widetilde{u} \widetilde{d} & \widetilde{d} & 0
\end{array}\right],
$$

where

$$
\begin{gathered}
\widetilde{u}=\frac{\sqrt{h_{R}} u_{R}+\sqrt{h_{L}} u_{L}}{\sqrt{h_{R}}+\sqrt{h_{L}}}, \quad \widetilde{h}=\frac{1}{2}\left(h_{R}+h_{L}\right), \quad \widetilde{c}=\sqrt{g \widetilde{h}}, \\
\widetilde{d}=\frac{A_{g} \xi\left(\sqrt{h_{R}}+\sqrt{h_{L}}\right)}{\sqrt{h_{L}} h_{R}+\sqrt{h_{R}} h_{L}} \sum_{k=0}^{m_{g}-1}\left(u_{R}\right)^{k}\left(u_{L}\right)^{m_{g}-(k+1)} .
\end{gathered}
$$

\subsection{Approximation of Roe matrix}

In the previous subsection we have presented a Roe matrix corresponding to Grass model. But for the other models considered in this work, is not always possible to obtain an explicit formula and its implementation is very costly. So, in practice we use the following approximation of Roe matrix 


$$
\mathcal{A}_{\Psi}\left(W_{L}, W_{R}\right)=\left[\begin{array}{ccc}
0 & 1 & 0 \\
-(\widetilde{u})^{2}+(\widetilde{c})^{2} & 2 \widetilde{u} & (\widetilde{c})^{2} \\
-\xi \frac{\partial q_{b}}{\partial h}(\widetilde{u}, \widetilde{h}) & -\xi \frac{\partial q_{b}}{\partial q}(\widetilde{u}, \widetilde{h}) & 0
\end{array}\right],
$$

with $\widetilde{u}, \widetilde{h}$ and $\widetilde{c}$ defined by (45), that is, the mean Roe values of $u, h$ and $c$ corresponding to Shallow Water equations.

Remark 3 We observe that as we use an approximated Roe matrix, we cannot use directly schemes on function of $\mathcal{A}^{ \pm}$, we must use the schemes that explicitly depend on the physical flux function $F(W)$, that is, scheme (36)-(37) or scheme (40)-(41).

\subsection{Numerical treatment of transitions close to the critical shear stress}

As we have seen in Section 2.3, in general, the movement of the sediment layer starts when shear stress $\tau$ is bigger than a critical shear stress $\tau_{c}$. So, when we use this type of models, at interface $x_{i+1 / 2}$, we could find some of the following situations:

st1) Transition with-without sediment: if $x_{i+1 / 2}$ is the interface between $I_{i}$ and $I_{i+1}$, with a sediment layer of thickness $z_{b, i}$ such that the shear stress at $I_{i}$ is bigger than the critical one $\left(\tau_{i}>\tau_{c}\right)$, and there is not sediment at the control volume $I_{i+1}$ (Figure $3(\mathrm{a})$ ).

st2) Transition with-without movement of the sediment: if $x_{i+1 / 2}$ is the interface between $I_{i}$ and $I_{i+1}$, with a sediment layer with thickness $z_{b_{i}}$ and such that the shear stress is bigger than the critical one $\left(\tau_{i}>\tau_{c}\right)$; and the volume $I_{i+1}$, with a sediment layer of thickness $z_{b, i+1}$ where the shear stress is smaller than the critical one $\left(\tau_{i+1}<\tau_{c}\right)$ (Figure 3(b)).

In order to avoid instabilities, it is necessary to change the numerical scheme so that these situations are numerically well treated. $3)$ :

For example, Let us suppose that in the intercell $x_{i+1 / 2}$ we have the following situation (Figure

$$
\begin{aligned}
& \tau_{i}>\tau_{c}, \\
& \tau_{i+1}<\tau_{c}, \\
& \tau_{i+1 / 2}<\tau_{c} ;
\end{aligned}
$$

where $\tau_{i}$ is the shear stress at volume $I_{i}$ and $\tau_{i+1 / 2}$ is the shear stress corresponding to the mean value of Roe. In this case, we propose the following algorithm:

1. $\mathcal{A}_{i+1 / 2}=\mathcal{K}_{i+1 / 2} \mathcal{L}_{i+1 / 2} \mathcal{K}_{i+1 / 2}^{-1}$.

2. Let $\lambda_{3}^{i+1 / 2}$ be the characteristic velocity corresponding to the sediment transport at the intercell. As $\tau_{i+1 / 2}<\tau_{c}$, then $\lambda_{3}^{i+1 / 2}=0$. Next, we set $\bar{\lambda}_{3}^{i+1 / 2}=\lambda_{3}^{i}$, where $\lambda_{i}^{3}$ is the characteristic velocity corresponding to the sediment transport at the cell $I_{i}$. Note that $\lambda_{i}^{3} \neq 0$ as $\tau_{i}>\tau_{c}$.

3. The diffusion matrix $\mathcal{D}_{i+1 / 2}$ is computed using the modified value $\bar{\lambda}_{3}^{i+1 / 2}$ instead of $\lambda_{3}^{i+1 / 2}=0$.

The algorithm can be summarized as follows: The values $\tau_{i}, \tau_{i+1}$ and $\tau_{i+1 / 2}$ are computed. If in the three cases the shear stress is bigger than the critical shear stress we do not perform any change. In the case of a transition we change the diffusion matrix as described before. 


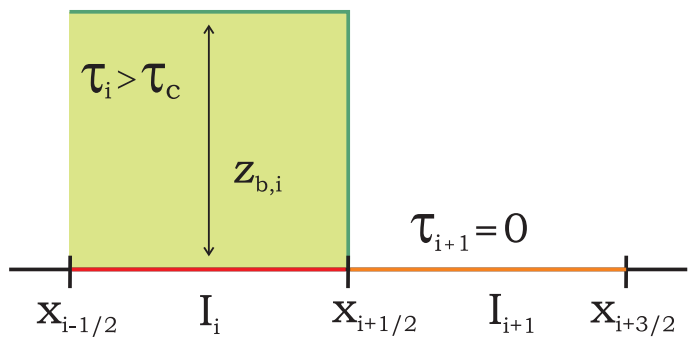

(a) With/without sediment.

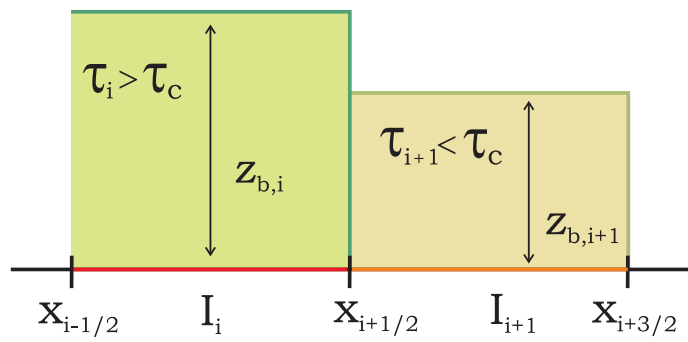

(b) With sediment in both cells, $\tau_{i}>\tau_{c}$ in cell $I_{i}$ and $\tau_{i+1}<\tau_{c}$ in cell $I_{i+1}$.

Figure 3: Possible cases of transition around the critical shear stress.

\section{$6 \quad$ Numerical test}

In this section we present some numerical tests to validate numerical schemes described in sections 3 and 4. We have considered an academic test with a known analytical solution, a test of order and an experimental test where we compare with experimental measures.

\subsection{Transport of parabolic sediment layer}

This first test consists on comparing a numerical solution with an asymptotic and analytical solution obtained by Hudson and Sweby in [31], for Grass model when interaction constant $A_{g}$ (8) is smaller than $10^{-2}$. In this case, the layer sediment $\tilde{z}_{b}$ is over all computational domain and fluid is moving slowly with a constant discharge $q=q_{0} \leq 10$. Under these hypothesis it is possible to obtain the following analytical solution,

$$
h=A_{r}-z_{b}(x, t), \quad q=q_{0}
$$

being $A_{r}$ a fixed level of reference, $q_{0} \leq 10$ a constant value, and

$$
z_{b}(x, t)= \begin{cases}\sin ^{2}\left(\frac{\pi\left(x_{o}-300\right)}{200}\right) & \text { if } 300 \leq x_{o} \leq 500 \\ 0 & \text { otherwise }\end{cases}
$$

where $x_{o}$ is the solution of equation

$$
\begin{cases}x=x_{o}+A_{g} \xi m_{g} q_{0}^{m_{g}} t\left(A_{r}-\sin ^{2}\left(\frac{\pi\left(x_{o}-300\right)}{200}\right)\right)^{-\left(m_{g}+1\right)} & \text { if } 300 \leq x_{o} \leq 500 \\ x=x_{o}+A_{g} \xi m_{g} q_{0}^{m_{g}} t A_{r}^{-\left(m_{g}+1\right)} & \text { otherwise. }\end{cases}
$$

The usual value of $m_{g}=3$ is considered.

This solution is valid to $t<t_{0}$, being $t_{0}$ the instant in which characteristics cross. In [31] it is estimated $t_{0}=238079.124 \times A_{g}^{-1}$.

In the same way, it is verified that

$$
\tilde{z}_{b}(x, t)=z_{b}(x, t)+c_{0},
$$

where $c_{0}$ is a constant value, it is also a solution of the problem. 


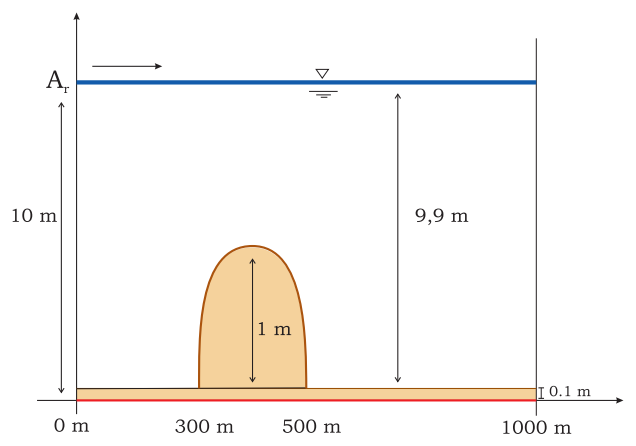

Figure 4: Initial condition.

To be able to compare numerical solution with analytical solution obtained in [31], without considering the behavior of numerical scheme in zones with-without sediment, we have compared the solution for the case,

$$
\tilde{z}_{b}(x, t)=z_{b}(x, t)+0.1 .
$$

We consider a computational domain whose length is $L=1000$ meters, discretized with 250 nodes. CFL condition is set to 0.8 . The sediment porosity is set to $\rho_{0}=0.4$ and the constant $A_{g}$ of Grass formula (8) is set to 0.001 which corresponds to a weak interaction. The initial conditions are (see Figure 4),

$$
\begin{gathered}
h(x, 0)=10-z_{b}(x, 0), \quad q(x, 0)=10, \\
z_{b}(x, 0)= \begin{cases}0.1+\sin ^{2}\left(\frac{\pi(x-300)}{200}\right) & \text { if } 300 \leq x \leq 500, \\
0.1 & \text { otherwise. }\end{cases}
\end{gathered}
$$

As boundary condition, the flux and the depth of the sediment is imposed upstream, while free boundary conditions are imposed downstream.

In Figures 5, 6, 7 we compare analytical solution (continuous line) and numerical solution obtained with the different schemes of sections 3 and 4 , corresponding to sediment layer evolution at instant $t=238080 \mathrm{~s}$; that is smaller than the maximal time in which analytical solution (50) is valid.

We can observe that all numerical schemes show a good sediment layer localization, being Roe scheme the most diffusive (Figure 5(a)). Moreover, the scheme that gives the best approximation is high order generalized Roe scheme with Weno state reconstructions of order 3, where time approximation is made using Runge-Kutta of order 3(Figure 7(a)).

In Figure 5 it is shown comparison between Roe method and linearized Lax-Wendroff method with flux limiters. In both methods we use Euler scheme for time discretization. In Figure 5(a), that describes layer sediment evolution, it is observed that Roe method is more diffusive than method with flux limiters, that is of second order in space and time for linear problems. In Figure 5(b) the discharge is presented. We observe that it is near 10 in both cases and almost constant in all domain, that is one of the hypothesis used to develop the analytical solution (50).

In Figure 6 it is compared flux limiter scheme with Roe-Weno of order 2. For time evolution it is used Euler for flux limiter scheme, because Lax-Wendroff scheme ensures order 2 in space and time. For scheme with Weno2 state reconstructions it is used TVD Runge-Kutta method of order 2. It must be observed that approximation of thickness sediment layer is similar in both schemes.

Finally in Figure 7 schemes with Weno2 and Weno3 state reconstructions are compared. For time evolution it is used Runge-Kutta2 and Runge-Kutta3, respectively. Higher order scheme approximates better the sediment layer thickness as expected. 


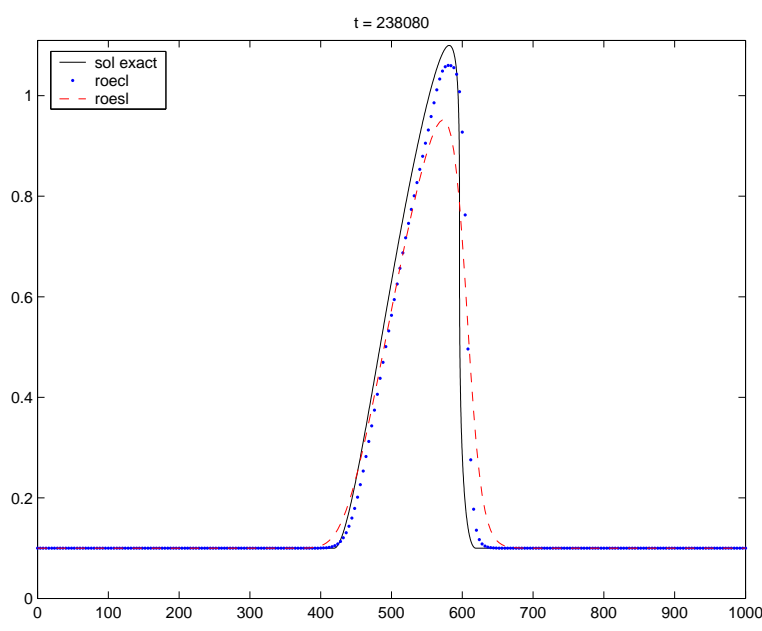

(a) Sediment layer thickness.

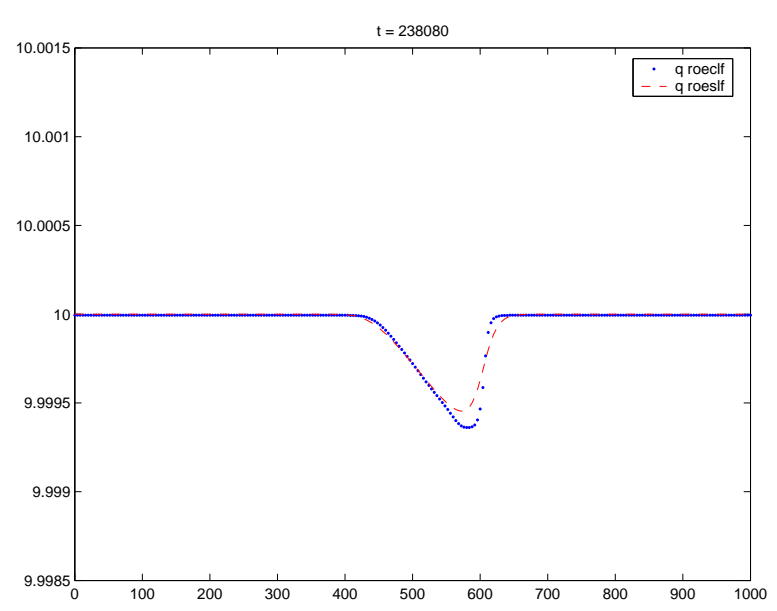

(b) Mass-flow.

Figure 5: Roe-Flux limiters (dotted line). Euler-Roe (dash line).

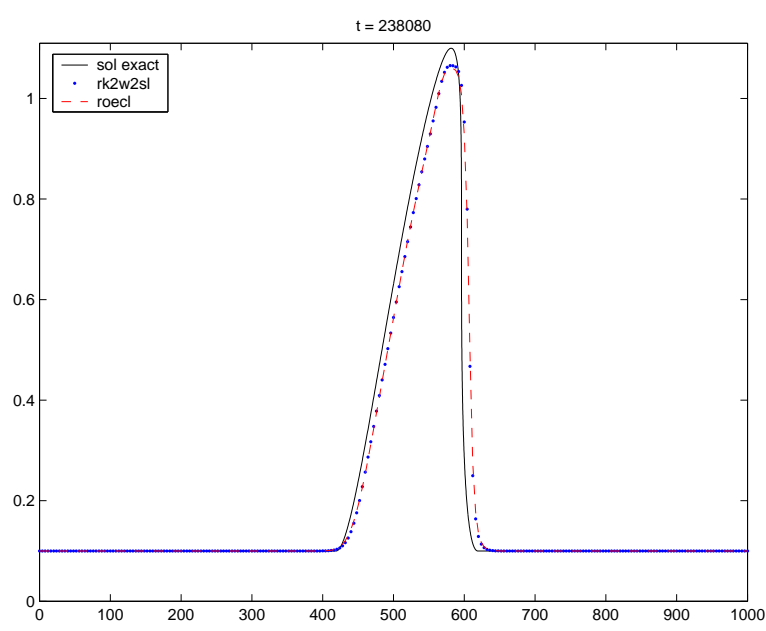

(a) Sediment layer thickness.

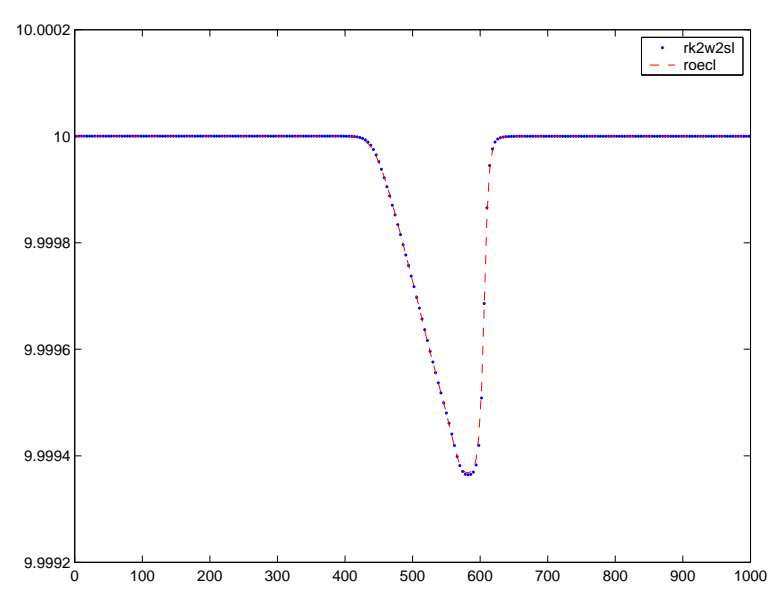

(b) Mass-flow fluid.

Figure 6: Roe-Flux limiters (dash line). Weno2-Rk2 (dotted line). 


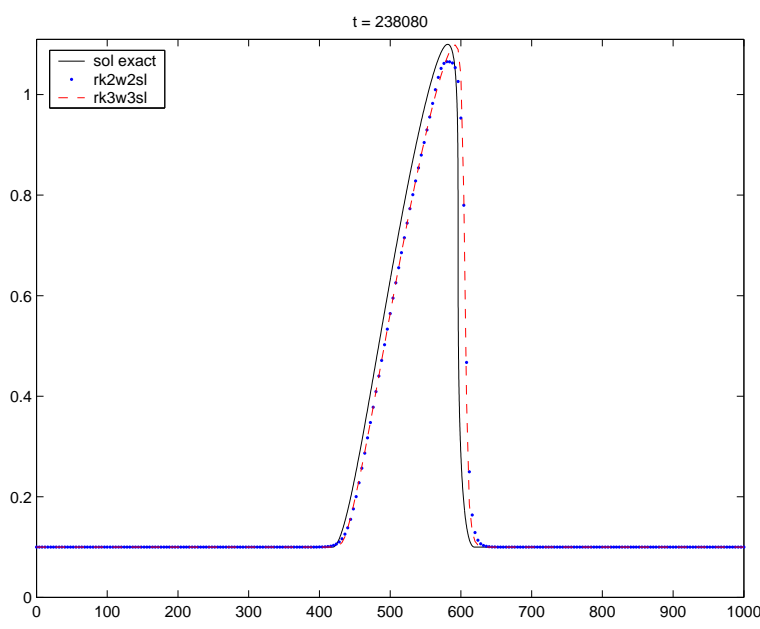

(a) Sediment layer thickness.

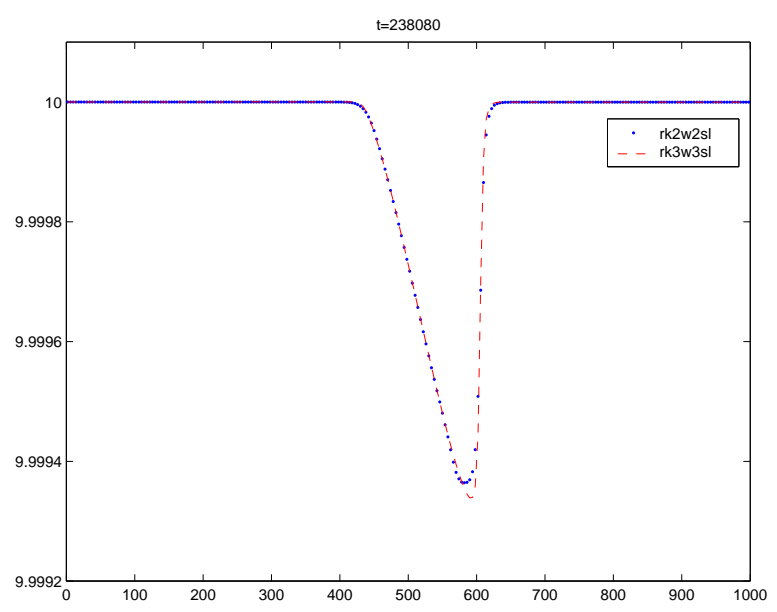

(b) Mass-flow fluid.

Figure 7: Weno2-Rk2 (dotted line). Weno3-Rk3 (dash line).

\subsection{Test of order}

We make the following test to compare the order of the proposed numerical schemes. We consider a one dimensional channel with flat bed along the interval $[-10,10]$, and with the following initial condition (see Figure 8),

$$
q(x, 0)=0, \quad h(x, 0)=2-0.1 e^{-x^{2}}, \quad z_{b}(x, 0)=0.1-0.01 e^{-x^{2}} .
$$

The exact solution for this problem is unknown, therefore we use as reference solution a numerical one obtained with a very fine mesh, of 5120 volumes.

This test has been made using Grass model (8) for solid discharge. We consider a strong interaction between sediment layer and fluid, concretely we impose $A_{g}=0.3$.

In Figures 9 and 10 the different results are presented, at instant $t=0.5 \mathrm{~s}$, obtained with the schemes: Euler-Roe (described in Section 3), Roe-Weno2-RungeKutta2, Roe-Weno3-RungeKutta3 (described in Section 4). Tables 2, 3 and 4 shown the errors for variables $h, q$ and $z_{b}$ in $L^{1}$ norm, for $t=0.5$. It must be observed that numerical schemes get in this test the expected order: Euler-Roe order one, Roe-Weno2-RungeKutta2 order two and Roe-Weno3-RungeKutta3 order three.

\begin{tabular}{|c|c|c|c|c|c|c|}
\hline Nodes & $L^{1}$ err $h$ & $L^{1}$ order $h$ & $L^{1}$ err $q$ & $L^{1}$ order $q$ & $L^{1}$ err $z_{b}$ & $L^{1}$ order $z_{b}$ \\
\hline \hline 20 & 0.1070 & & 0.4118 & & $0.6148 \times 10^{-3}$ & \\
\hline 40 & 0.0701 & 0.6111 & 0.2810 & 0.5513 & $0.4218 \times 10^{-3}$ & 0.5433 \\
\hline 80 & 0.0418 & 0.7441 & 0.1733 & 0.6971 & $0.2726 \times 10^{-3}$ & 0.6297 \\
\hline 160 & 0.0234 & 0.8370 & 0.0985 & 0.8154 & $0.1639 \times 10^{-3}$ & 0.7335 \\
\hline 320 & 0.0124 & 0.9066 & 0.05287 & 0.8977 & $0.1639 \times 10^{-3}$ & 0.85430 \\
\hline 640 & 0.0064 & 0.9521 & 0.0274 & 0.9481 & $0.0907 \times 10^{-3}$ & 0.9168 \\
\hline
\end{tabular}

Table 2: Errors and order: generalized Roe scheme. 


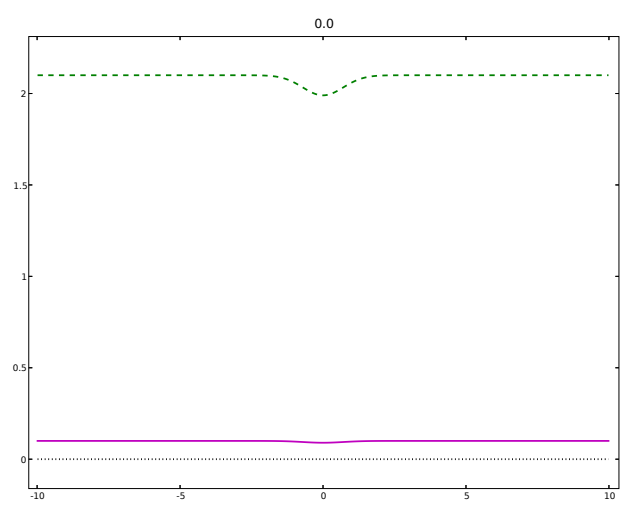

(a) Initial condition

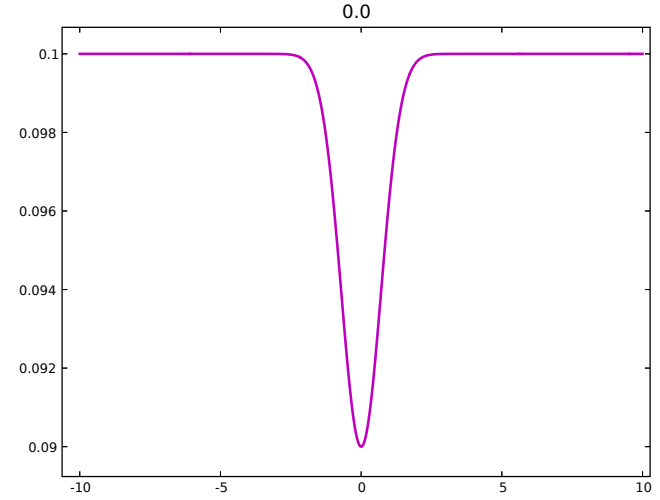

(b) Sediment layer thickness $z_{b}$.

Figure 8: Test of order. Initial condition. Layer water thickness $(h)$ : dashed line. Sediment layer thickness $\left(z_{b}\right)$ : continuous line. Depth $(H)$ : dotted line.

\begin{tabular}{|c|c|c|c|c|c|c|}
\hline Nodes & $L^{1}$ err $h$ & $L^{1}$ order $h$ & $L^{1}$ err $q$ & $L^{1}$ order $q$ & $L^{1}$ err $z_{b}$ & $L^{1}$ order $z_{b}$ \\
\hline \hline 20 & 0.0968 & & 0.4050 & & $0.5338 \times 10^{-3}$ & \\
\hline 40 & 0.0521 & 0.8923 & 0.2226 & 0.8630 & $0.3073 \times 10^{-3}$ & 0.7966 \\
\hline 80 & 0.0160 & 1.7043 & 0.0689 & 1.6919 & $0.1331 \times 10^{-3}$ & 1.2074 \\
\hline 160 & 0.0047 & 1.7515 & 0.0203 & 1.7580 & $0.0418 \times 10^{-3}$ & 1.6702 \\
\hline 320 & 0.0012 & 2.0000 & 0.0051 & 2.0025 & $0.0113 \times 10^{-3}$ & 1.8824 \\
\hline 640 & 0.0003 & 2.0995 & 0.0012 & 2.0934 & $0.0027 \times 10^{-3}$ & 2.0303 \\
\hline
\end{tabular}

Table 3: Errors and order: Weno2 + Runge Kutta 2

\subsection{Comparison with experimental data}

In this section we present an experiment where we simulate sediment layer evolution over a soil which is not eroded. The numerical stationary solution is compared with the experimental data obtained by the Hydraulic Laboratory of Escuela Superior de Ingenieros de Caminos, Canales y Puertos (A Coruña University) over a channel of $15 \mathrm{~m}$ long and $0.5 \mathrm{~m}$ width.

E. Peña González details in his Phd Thesis [45] different techniques and toolkits used to develop this and other experiments. In this particular case, measures were made using a PIV technique and a Scanner 3D technique (in [45] the details of the different measurement techniques are explained).

The experimental test was developed introducing a sand layer in the central part of laboratory channel, and inducing hydrodynamical conditions to erode the sand layer, until a steady state is reached. The channel (Figure 11 ) has a very small slope of $0.052 \%$. Sand layer was situated in interval $[4.5 \mathrm{~m}, 9 \mathrm{~m}$ ], with a thickness of $4.5 \mathrm{~cm}$; being media diameter of the grain equals to $1 \mathrm{~mm}$. During the experiment the sand layer was eroded very slowly, extracting material from upstream at same time that sand was being transport downstream, creating a tongue of sand.After approximately 8 hours, a stationary state is reached and the sand front was placed at the point $x_{s}=12.15 \mathrm{~m}$, so the total material advance was $3.15 \mathrm{~m}$. The final thickness of the sand layer was approximated equal to $3 \mathrm{~cm}$ along all length, except in the transition areas at initial and final extremes.

The numerical simulation has been made using Meyer-Peter\&Müller's equation (10) for solid transport discharge. 


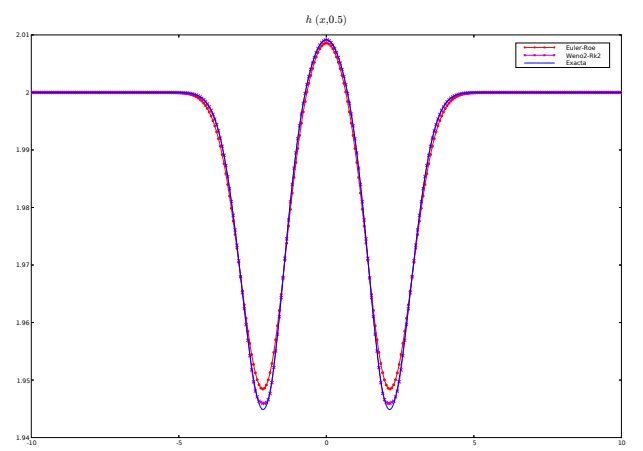

(a) Water layer thickness $h$.

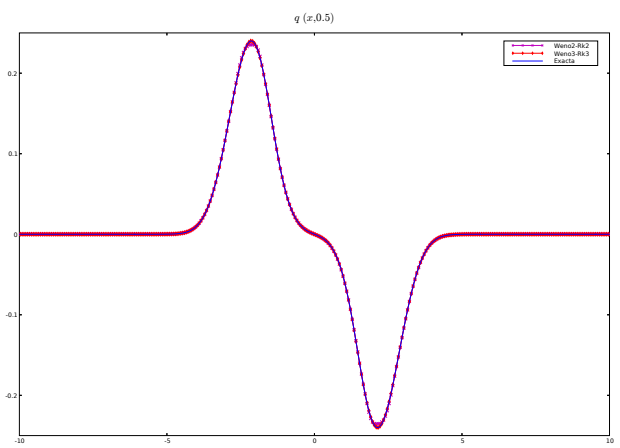

(b) Mass-flow $q$.

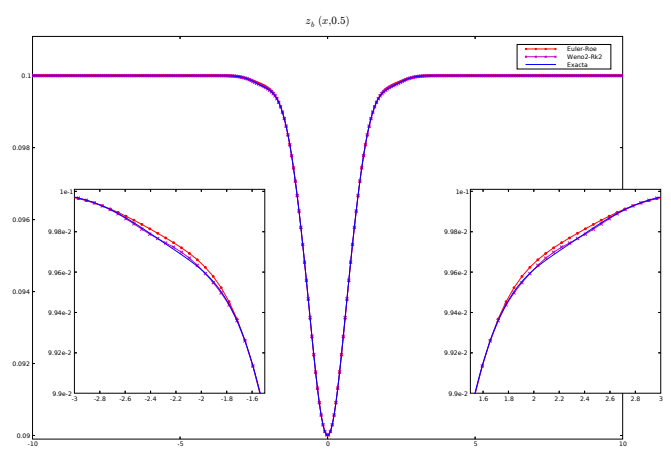

(c) Sediment layer thickness $z_{b}$.

Figure 9: Order test. Comparison between the solution at $t=0.5 \mathrm{~s}$. Fine mesh (continuous line). Euler-Roe (continuous line with dots). Weno2-Rk2 (continuous line with stars).

The first problem is to give an admissible initial condition for the laboratory experiment, because we only know the position and the thickness of the sediment layer $z_{b}$ at the steady state, but we have not information about initial conditions. To build an initial condition we have proceeded as follows:

a) Initial condition for flux: we know by experimental data that the average thickness of the sediment layer at the steady state is about $3 \mathrm{~cm}$. Moreover the thickness of the water column over the sediment layer is approximately $9.5 \mathrm{~cm}$. Using the shear stress expression and the critical shear stress value, it is possible to determine the critical discharge value, $q_{c}$, for which solid transport discharge is zero. We obtain the value of $q_{c}=0.0285 \mathrm{~cm}^{2} / \mathrm{s}$, which we use as initial condition for the flux.

b) As we have information about the thickness of the water layer at the right extreme of the channel, and therefore, fluid surface elevation, $\bar{\eta}$, a first choice for the initial condition over the thickness of the water column is to consider $h(x, 0)=\bar{\eta}-H(x)-z_{b}(x, 0)$. However, for this initial condition we observe an 'anomalous' behavior of the evolution of the sediment layer. To avoid this problem, firstly we calculate the free water surface using Bernoulli's equation, imposing a constant discharge $q=q_{c}$ along the channel and using as topography the function 


\begin{tabular}{|c|c|c|c|c|c|c|}
\hline \multirow{2}{*}{ Nodes } & err & order & err & order & err & order \\
& $L^{1} h$ & $L^{1} h$ & $L^{1} q$ & $L^{1} q$ & $L^{1} z_{b}$ & $L^{1} z_{b}$ \\
\hline \hline 20 & 0.0398 & & 0.2690 & & $0.2689 \times 10^{-3}$ & \\
\hline 40 & 0.0115 & 1.7912 & 0.0479 & 2.4880 & $0.0656 \times 10^{-3}$ & 2.0333 \\
\hline 80 & 0.0009 & 3.6751 & 0.0038 & 3.6307 & $0.0064 \times 10^{-3}$ & 3.3407 \\
\hline 160 & $6.0 \times 10^{-5}$ & 3.8304 & 0.00026 & 3.858 & $4.5709 \times 10^{-7}$ & 3.8264 \\
\hline 320 & $6.0 \times 10^{-6}$ & 3.3870 & $2.5334 \times 10^{-5}$ & 3.3973 & $4.2298 \times 10^{-8}$ & 3.4338 \\
\hline 640 & $6.9 \times 10^{-7}$ & 3.1220 & $2.9077 \times 10^{-6}$ & 3.1231 & $4.8039 \times 10^{-9}$ & 3.1382 \\
\hline
\end{tabular}

Table 4: Errors and order: Weno3 + Runge Kutta 3.

given by $\bar{H}(x)=H(x)-z_{b}(x, 0)$. That is, calculating the steady solution for the topography $\bar{H}(x)=H(x)-z_{b}(x, 0)$, the discharge $q=q_{c}$, and such that the thickness of the water layer at the right extreme of the channel is equal to the experimental measure.

Therefore, as we do not know the initial condition and we must impose it using the technique explained before, we can only compare the numerical and experimental solution at steady state. In Figure 12 we show the initial condition used in this numerical simulation.

For numerical simulation we have meshed the domain with 250 nodes The CFL is set to 0.9. Sediment porosity is set to 0.4. Friction between fluid and bed is modeled using a Manning's law with coefficient equal to 0.0125 over the fixed bed and 0.0196 over the sediment layer. As boundary conditions we impose an incoming discharge equal to $0.0285 \mathrm{~m}^{2} / \mathrm{s}$ upstream, while the water thickness is imposed to $0.129 \mathrm{~m}$ downstream.

The model that we are using does not include pressure forces, so the sediment does not fall by its own weight due to gravity effects. For this reason we cannot expect that the numerical scheme could reproduce accurately the zones where gravity effects are relevant, as for example, the advancing front of the sand layer, but it must reproduce at least, the downstream sand slope and the median profile of the sediment layer. This behavior is reflected in Figure 13, that shows at 10 min, 40 min y 120 min, comparison between experimental measures (line with dots) obtained by a classic technique (see [45]), and the numerical solutions obtained with Roe scheme without state reconstructions (dash line) and scheme with Weno2 state reconstructions (continuous line). In this figure it is possible to observe that the scheme of order one (Roe without state reconstructions) is more diffusive than the scheme of order two. Although we do not know the initial condition, the numerical solution tends to the steady state. In the different instants, the graphics show that we are approximating the average of the thickness sediment layer. Moreover, numerically it is proved that we conserve total mass of sediment layer.

In Figure 14 we compare the experimental measures of the position of the sediment layer top at the steady state with the numerical solutions obtained using different numerical schemes. In this figure line with dots represents experimental data, line with squares represents the solution obtained by scheme with reconstruction Weno2, and line with diamonds represents the solution got by Roe scheme without state reconstructions. In Figure 14 it is observed that both numerical solutions converge slowly to the steady state.

In this example, it is difficult to say what numerical scheme reproduce better the laboratory experiment, mainly because the physical model does not include pressure forces, so the sediment does not fall by its own weight due to gravity effects. Nevertheless, we could say that, in general, (see Figure 13), the numerical scheme Rk2-Weno2 captures better the depth profiles of the sediment layer at the initial part of the sediment layer, where gravity effects are not so relevant, while Euler-Roe scheme not. A more complex physical model including the gravity effects at the sediment layer must be considered in order to reproduce better the laboratory experiment. 


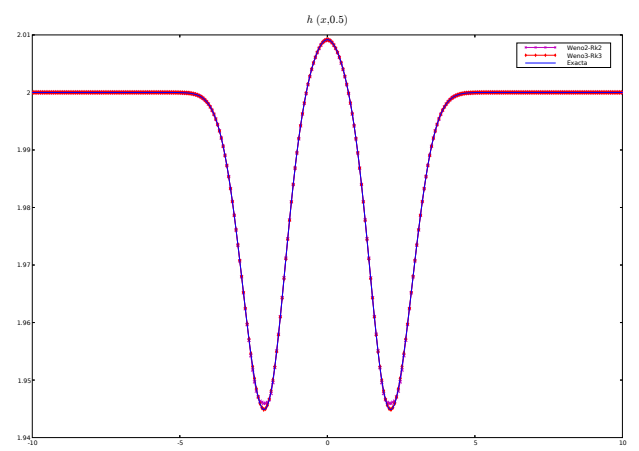

(a) Water layer thickness $h$

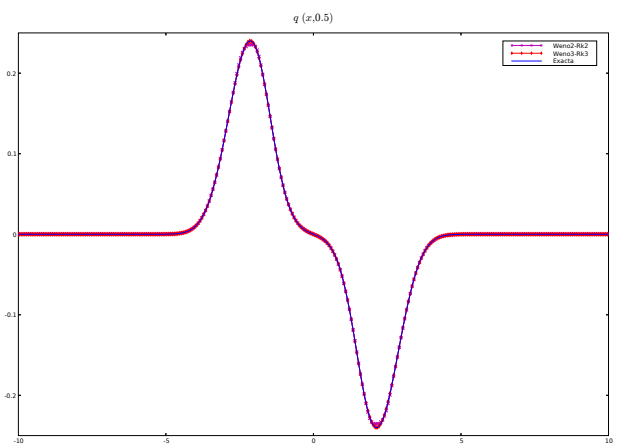

(b) Mass-flow $q$.

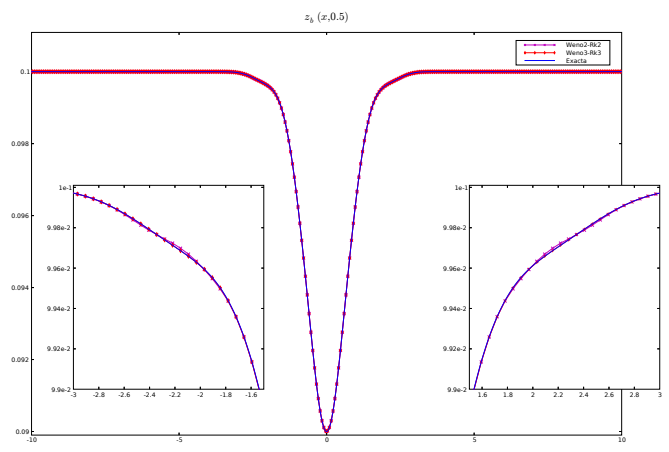

(c) Sediment layer thickness $z_{b}$.

Figure 10: Order test. Comparison with the fine mesh solution in $t=0.5 \mathrm{~s}$. Fine mesh solution (continued line). Weno2-Rk2 (continued line with stars). Weno3-Rk3 (continued line with diamonds)

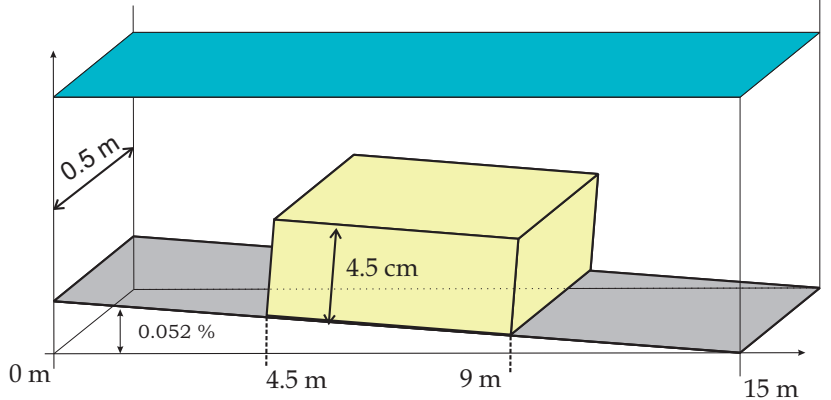

Figure 11: Sketch of the laboratory experiment.

\section{References}

[1] F. Alouges, B. Merlet. Approximate shock curves of non-conservative 


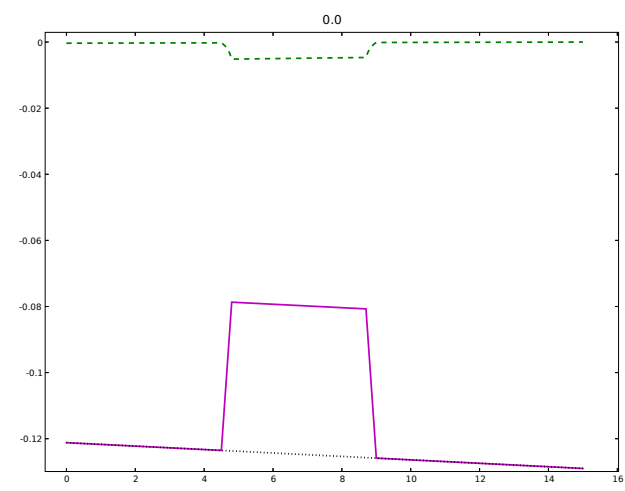

Figure 12: Initial condition imposed in numerical test. Layer water thickness $(h)$ : dashed line. Sediment layer thickness $\left(z_{b}\right)$ : continuous line. Depth $(H)$ : dotted line.

hyperbolic equations in one space dimension. Preprint, available at http://mahery.math.u-psud.fr/ alouges/full_publi.html.

[2] F. Alcrudo, F. Benkhaldoun. Exact solutions to the Riemann problem of the shallow water equations with a bottom step, Computer \& Fluids 30: 643-671, 2001.

[3] E. Audusse, F. Bouchut, M.O. Bristeau, R. Klein, B. Perthame. A fast and stable wellbalanced scheme with hydrostatic reconstruction for shallow water flows, SIAM J. Sci. Comp. 25 (6): 2050-2065, 2004.

[4] A. Bermúdez, M.E. VÁzquez. Upwind methods for hyperbolic conservation laws with source terms. Computers and Fluids, 23(8): 1049-1071, 1994.

[5] S. Bianchini, A. Bressan. Vanishing viscosity solutions of nonlinear hyperbolic systems. Annals of Mathematics, 161(1): 223-342, 2005.

[6] F. Bouchut. Nonlinear Stability of Finite Volume Methods for Hyperbolic Conservation Laws and Well-Balanced Schemes for Sources. Birkhäuser, 2004.

[7] F. Bouchut, F. JAmes. One-dimensional transport equations with discontinuous coefficients. Nonlinear Anal. TMA, 32, 891-933, 1998.

[8] Pilar Brufau. Simulación bidimensional de flujos hidrodinámicos transitorios en geometrías irregulares. Tesis doctoral. Universidad de Zaragoza, 2000.

[9] M.J. Castro, J. Macías y C. Parés. A Q-Scheme for a class of systems of coupled conservation laws with source term. Application to a two-layer 1-D shallow water system. Math. Mod. Num. Anal. 35(1): 107-127, 2001.

[10] M.J. Castro, J.M Gallardo, C. Parés. Finite volume schemes based on WENO reconstruction of states for solving nonconservative hyperbolic systems. Applications to Shallow Water systems. Accepted on Math. Comp.

[11] J.J. Cauret, J.F. Colombeau, A.Y. LeRoux. Discontinuous generalized solutions of nonlinear nonconservative hyperbolic equations. J. Math. Anal. Applic. 139: 552-573, 1989.

[12] T. Chacón, A. Domínguez, E.D. Fernández. A family of stable numerical solvers for Shallow Water equations with source terms. Comp. Meth. Appl. Mech. Eng. 192: 203-225, 2003. 


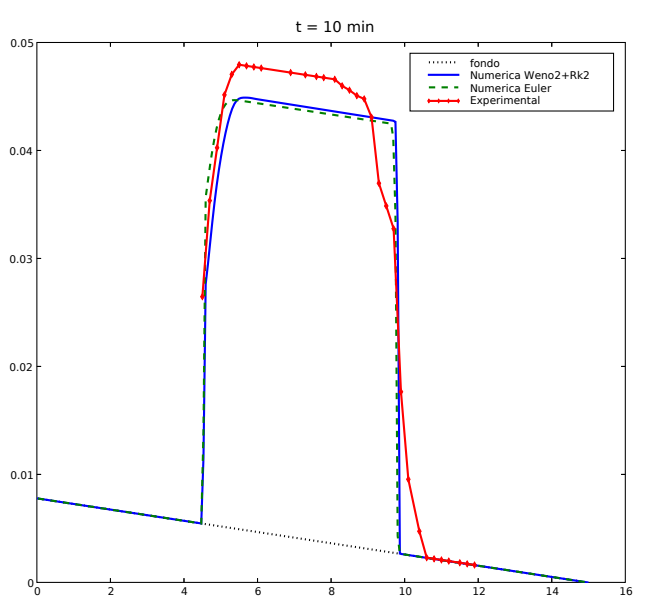

(a) 10 min.

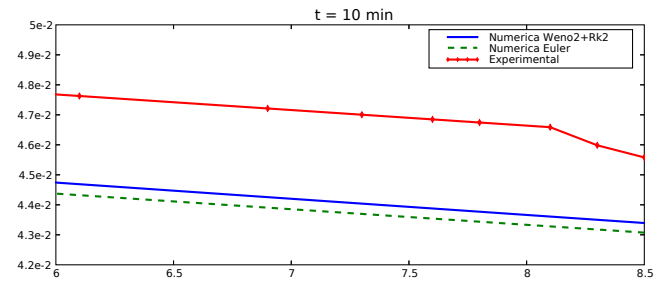

(c) 10 min. Detail of the sediment layer top

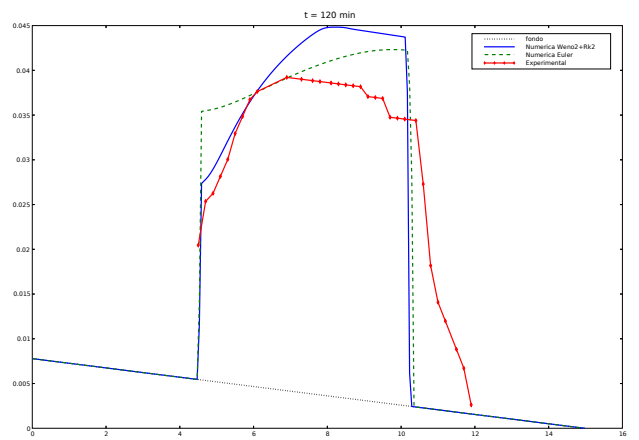

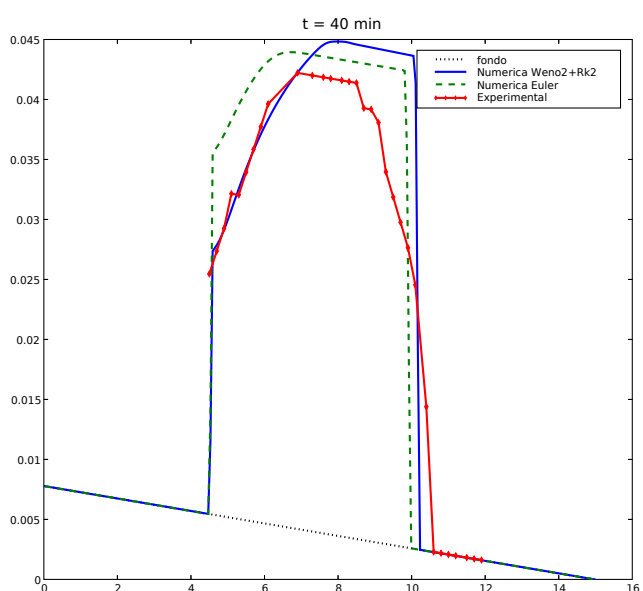

(b) $40 \mathrm{~min}$.

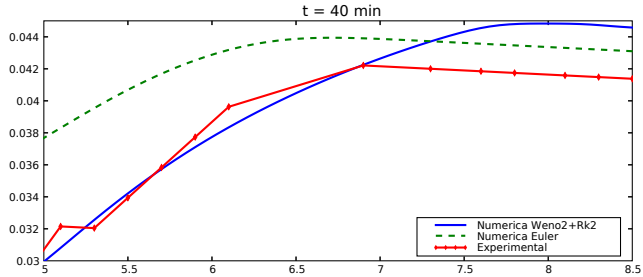

(d) 40 min. Detail of the sediment layer top

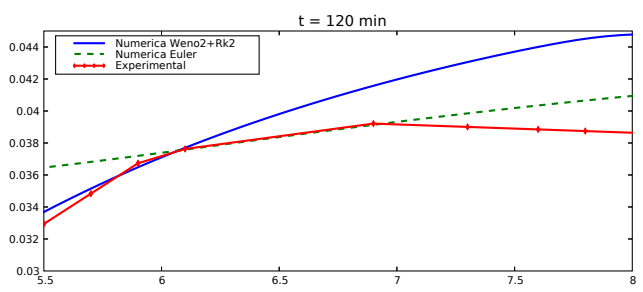

(f) 120 min. Detail of the sediment layer top

(e) $120 \mathrm{~min}$.

Figure 13: Sediment layer evolution at $10 \mathrm{~min}, 40 \mathrm{~min}$ y $120 \mathrm{~min}$. Experimental measures (continuous line with dots). Euler-Roe (dashed line). Weno2-Rk2 (continuous line). 


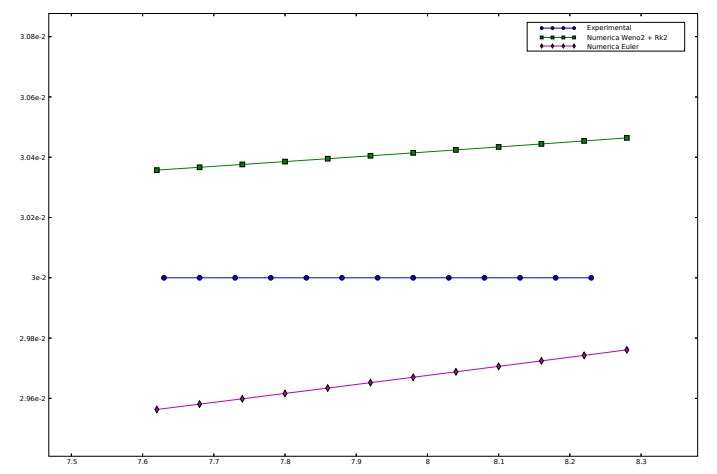

Figure 14: Comparison of the stationary solution for the sediment layer at the interval $[7.6 \mathrm{~m}, 8.5 \mathrm{~m}]$. Experimental data (line with points). Euler-Roe (line with rhombus) Rk2-Weno2 method (line with squares).

[13] T. Chacón, A. Domínguez, E.D. Fernández. Asymptotically balanced schemes for non-homoegeneous hyperbolic systems - application to the Shallow Water equations. C.R. Acad. Sci. Paris, Ser. I 338: 85-90, 2004.

[14] N. ChIEn. The present status of research on sediment transport. Trans. ASCE, 121S: 833-68; 1956.

[15] J.F. Colombeau, A. Heibig. Nonconservative products in bounded variation functions. SIAM J. Math. Anal. 23, 4:941-949, 1992.

[16] G. Dal Maso, P.G. LeFloch, F. Murat. Definition and weak stability of nonconservative products. J. Math. Pures Appl. 74: 483-548, 1995.

[17] Duboys, E. Meyer-Peter. Etudes du régime du Rhone et de l'action exercé par les eaux sur un lit à fond de graviers indéfiniment affouillable. Ann. Ponts et Chaussées, ser. 5, 18: 141-195, 1979.

[18] H.A. Einstein Formulas for the transportation of bed load. Trans. ASCE. 107: 561-575, 1942.

[19] H.A. Einstein The bed load function for sediment transport in open channel flows. Technical Bulletin n0. 1026. Washington, D.C.: U.S. Department of Agriculture, Soil Conservation Service. 1950 .

[20] L. Fracarollo, H. Capart and Y. Zech. A Godunov method for the computation of erosional shallow water transients. Int. J. Numer. Meth. Fluids 41:951-976, 2003.

[21] E. Godlewski, P.A. Raviart. Numerical Approximation of Hyperbolic Systems of Conservation Laws. Springer-Verlag, New York, 1996.

[22] L. Gosse. A well-balanced flux-vector splitting scheme designed for hyperbolic systems of conservation laws with source terms. Comp. Math. with Applic. 39:135-159, 2000.

[23] L. Gosse. A well-balanced scheme using non-conservative products designed for hyperbolic system of conservation laws with source terms. Mat. Mod. Meth. Appl. Sc. 11: 339-365, 2001.

[24] S. Gottlieb, C.W Shu. Total variation diminishing Runge-Kutta schemes. Math. Comp. 67: 73-85, 1998. 
[25] A.J. Grass. Sediments transport by waves and currents. SERC London Cent. Mar. Technol., Report No. FL29, 1981.

[26] J.M. Greenberg, A.Y. LeRoux. A well balanced scheme for the numerical processing of source terms in hyperbolic equations. SIAM J. Numer. Anal. 33: 1-16, 1996.

[27] J.M. Greenberg, A.Y. LeRoux, R. Baraille, A. Noussair. An Introduction to Continuum Mechanics. Academic Press, 1981.

[28] A. Harten. On a class of high resolution total-variation-stable finite-difference schemes. SIAM J. Numer. Anal. 21(1): 1-23, 1984.

[29] A. Harten, J.M. Hyman. Self-adjusting grid methods for one-dimensional hyperbolic conservation laws. J. Comp. Phys. 50: 235-269, 1983.

[30] Changqing Hu, C.W. Shu. Weighted essentialy non-oscillatory schemes on triangular meshes. J. Comput. Phys. 150: 97-127, 1999.

[31] J. Hudson. Numerical technics for morphodynamic modelling. Tesis doctoral. University of Whiteknights, 2001.

[32] G. Jiang, C.W. Shu. Efficient implementation of wieghted ENO schemes. J. Comput. Phys. 126: 202-228, 1996.

[33] P.Y. Julien. Erosion and Sedimentation. Cambridge Unilversity Press. 1998.

[34] A.A. Kalinske Criteria for determining sand transport by surface creep and saltation. Trans. AGU. 23(2): 639-643, 1942.

[35] A.A. Kalinske Movement of sediment as bed load in rivers. Trans. AGU. 28(4): 615-620, 1947.

[36] A. Kurganov, D. Levy. Central-Upwind schemes for the Saint-Venant System, Math. Mod. Num. Analysis, 36, 3997-425, 2002.

[37] P.G. LeFloch. Propagating phase boundaries; formulation of the problem and existence via Glimm scheme. Arch. Rat. Mech. Anal. 123: 153-197, 1993.

[38] P.G. LeFloch, A.E. Tzavaras. Representation and weak limits and definition of nonconservative products. SIAM J. Math. Anal. 30: 1309-1342, 1999.

[39] R. LeVeque. Numerical methods for conservations laws. Birkhauser Verlag, Zurich, 1990.

[40] R. LeVeque. Balancing source terms and flux gradients in high-resolution Godunov methods: the quasi-steady wave-propagation algorithm. J. Comp. Phys. 146: 346-365, 1998.

[41] E. Meyer-Peter, R. Müller. Formulas for bed-load transport. Rep. 2nd Meet. Int. Assoc. Hydraul. Struct. Res., Stockholm: 39-64, 1948.

[42] P. Nielsen. Coastal Bottom Boundary Layers and Sediment Transport. World Scientific Publishing, Singapore, Advanced Series on Ocean Engineering, 4; 1992.

[43] C. Parés, M.J. Castro. On the well-balanced property of Roe method for nonconservative hyperbolic systems. Applications to shallow water systems. ESAIM: M2AN, Vol 38(5): 821-852, 2004.

[44] C. Parés Numerical methods for nonconservative hyperbolic systems: a theoretical framewor. Accepted on SINUM. 
[45] Enrique Peña González. Estudio numérico y experimental del transporte de sedimentos en cauces aluviales. Tesis Doctoral. Universidade da Coruña. Grupo de Ingeniería del agua y del medio ambiente. 2002.

[46] B. Perthame, C. Simeoni. A kinetic scheme for the Saint-Venant system with a source term. Calcolo 38(4): 201-231, 2001.

[47] B. Perthame, C. Simeoni. Convergence of the upwind interface source method for hyperbolic conservation laws. Proc. of Hyp 2002. Thou and Tadmor ed. Springer, 2003.

[48] J.P. RAYMond. A new definition of nonconservative products and weak stability results. Boll. Un. Mat. Ital. B(7) 10, n. 3: 681-699, 1996.

[49] E.V. Richardson, D.B. Simons, P.Y. Julien. Hightways in the River Environment. Design and training manual for U.S. Department of Transportation, Federal Hightway Administration, publication no. FHWA-HI-90-016. Washington, D.C., 1990.

[50] P.L. RoE. Upwinding difference schemes for hyperbolic conservation laws with source terms. In Carasso, Raviart, and Serre, editors, Proceedings of the Conference on Hyperbolic Problems, 41-51. Springer, 1986.

[51] K. Sebastian, C.W. Shu. Multidomain WENO Finite Difference Method with Interpolation at Subdomain Interfaces. J. Scientific Comp. 19(1-3): 405-438, 2003.

[52] D.B. Simons, F. Senturk. Sediment Transport Technology. Littleton, Colo.: Water Resources Pub., 1977.

[53] C.W. SHu. Essentially non-oscillatory and weighted essentially non-oscillatory schemes for hyperbolic conservation laws. ICASE Report: 97-65, 1997.

[54] C.W. Shu, S. Osher. Efficient implementation of essentially non-oscillatory shock capturing schemes. J. Comput. Phys. 77: 439-471, 1998.

[55] R. Soulsby. Dynamics of marine sands. A manual for practical applications. Published by Thomas Telford Publications, Thomas Telford Services Ltd, 1997.

[56] E.F. Tono. Shock-Capturing Methods for Free-Surface Shallow Flows. Wiley, 2001.

[57] I. Toumi. A weak formulation of Roe approximate Riemann Solver. J. Comp. Phys. 102(2): 360-373, 1992.

[58] L.C. Van Risn. Sediment transport (I): bed load transport. J. Hydraul. Div., Proc. ASCE, 110: 1431-56, 1984.

[59] L.C. Van Risn. Sediment transport (II): suspended load transport. J. Hydraul. Div., Proc. ASCE, 111: 1613-41, 1984.

[60] L.C. Van Rijn. Sediment transport (III): bed forms and alluvial roughness. J. Hydraul. Div., Proc. ASCE, 112: 1733-54, 1984.

[61] M.E. Vázquez-Cendón. Estudio de Esquemas Descentrados para su Aplicación a las Leyes de Conservación Hiperbólicas con Términos Fuente. Tesis doctoral. Universidad de Santiago de Compostela, 1994.

[62] M.E. VÁzquez-CENDón. Improved treatment of source terms in upwind schemes for the shallow water equations in channels with irregular geometry. J. Comp. Phys. 148: 497-526, 1999.

[63] A.I. VolPert. The space BV and quasilinear equations. Math. USSR Sbornik 73(115): 225-267, 1967. 
[64] M.S. Yalin, E. Karahan. Inception of sediment transport. J. Hyd. Div. ASCE, 105(11): 133143, 1979.

[65] J.G. Zhou, D.M. Causon, C.G. Mingham, D.M. Ingram. The surface gradient method for the treatment of source terms in the shallow-water equations. J. Comp. Physics. 168: 1-25, 2001.

[66] italianos LHLL. 